\title{
Numerical Study of New Device for Passive Suppression of Vortex-Induced Vibrations in Deep Water Risers
}

Mohammed Abdulaziz ( $\square$ mohammed.abdulaziz@stud.uni-due.de)

University of Duisburg-Essen https://orcid.org/0000-0002-7050-033X

Emad Elsaid

Fayoum University Faculty of Engineering

Original Article

Keywords: VIV, Riser, FSI, OpenFOAM, PIMPLE, LES

Posted Date: June 9th, 2020

DOI: https://doi.org/10.21203/rs.3.rs-33449/v1

License: (c) (1) This work is licensed under a Creative Commons Attribution 4.0 International License.

Read Full License 


\title{
Numerical Study of New Device for Passive Suppression of Vortex- Induced Vibrations in Deep Water Risers
}

\author{
1 Mohammed Abdulaziz \\ 2 Emad M.S. El-Said* \\ 1 Institute of Ship Technology, Ocean Engineering and Transport Systems (ISMT), University of Duisburg-Essen, \\ Duisburg, Germany \\ Mohammed.abdulaziz@stud.uni-due.de \\ $2 *$ Department of Mechanical Engineering, Faculty of engineering, Fayoum University, Fayoum, Egypt. \\ emadsaad@fayoum.edu.eg
}

\begin{abstract}
This paper presents a three dimensional numerical simulation to investigate the effectiveness of a new passive device for vortex-induced vibrations (VIV) suppression of deep water risers based on non-continuous helical strakes in staggered arrangement with two starts. The influence of changing the geometries and design parameters, such as the pitch and shape of strakes, on the VIV was studied. The simulation had been carried out at Reynolds number 104, which satisfies the natural conditions of ocean currents. The presented design has been tested using a numerical solver using OpenFOAM platform, which had been presented and validated using number of published benchmarks. The LES - large-eddy simulation is used for turbulence modeling. The results had been compared with the published numerical and experimental works and other design based on drag coefficient. The comparison shows that the proposed geometry can suppress the VIV, if applied in deep water risers. The drag coefficient of 1.05 is considered a reduced value in the modified strakes shape with two starts and non-continuous helical strakes in staggered arrangement. The addition of strakes to the surface of a flexible cantilever was modeled to give the chance for evaluating the effect of geometry on the VIV.
\end{abstract}

Keywords: VIV; Riser; FSI; OpenFOAM; PIMPLE; LES

\section{Introduction}


The vortex induced vibration of deep water risers is one of the most critical and important issues in the field of ocean engineering. Although many research works had been carried out along past tens of years, many research points are still open.

In deep water, where the risers are usually working, the so-called deep water current is always going on with different speeds which can generate a water flow, which could be considered as highly turbulent [1]. When these deep water currents meet the risers, the so-called vortex shedding is formed around the riser itself [2]. This phenomenon results lift force unsteady oscillation, because pressure distribution unsteady changes on the riser surface, which directly causes an unsteady oscillating motion for the riser. This unsteady oscillating motion of the riser, or any obstacle in other cases, called VIV. Such motion is very usual resulting long-term harmful effects on the riser in deep water, which can lead to riser failure and eventually an environmental disaster. When the frequency of VIV approaches the natural frequency of the structure, a resonant phenomenon called lock-in is occurring.

\section{Literature Review}

Kim et al. [3] experimentally studied the flow-induced vibrations (FIV) of a circular cylinder attached by flexible rectangular sheets in the downstream direction, which were attached axially to the cylinder's surface to control flow-induced vibration. The sheets angle (between the global flow direction and a line connecting the attachment point to the center of the cylinder) varied between $90^{\circ}$ to $-90^{\circ}$. While both flexible sheet length and width of sheet varied between 0.5 to 3.0 times the cylinder's diameter and between 1.0 to and 0.4 times the cylinder length respectively. Their results showed a significant influence of the sheets angle on the ratio between vibration amplitude and cylinder diameter (y/D). The Reynolds number was 4365 to 74200 in this study. The optimal length and minimum width of the flexible sheet that could suppress the flowinduced vibration of the cylinder are 2 to 2.5 times the cylinder diameter and 0.7 times the cylinder length, respectively. Li et al. [4] experimentally studied the hydrodynamic forces for a VIV case of a flexible circular cylinder. Their model was a $2.6 \mathrm{~m}$ steel pipe positioned in a water tank. The mass ratio of this case was 2.62, while a Reynolds number range of $\operatorname{Re}<9600$ was considered. The gap ratios (gap to cylinder diameter ratio e/D) at the cylinder ends are 2.0, 4.0, 6.0 and 8.0. They performed a comparison to the published results and showed good agreement. The effect of gap ratio on the force and added mass coefficient is weak for e/D > 2.0, but a significant effect is shown for $\mathrm{e} / \mathrm{D}=2.0$. The study gives the chance for validation of 
computational work results for the same case. Xu et al. [5] carried out a numerical study with the help of experiments on the in-line VIV of the slender marine structure, in which only the in-line vibrations were considered. A wake oscillator model was developed to analyze an inline VIV of such slender marine structures. Moreover, two different kinds of van der Pol equations were used to describe the near wake dynamics. It was concluded that the presented model is not applicable in the case of nonuniform flows, while the comparison of results versus the experiments concluded that the presented model reproduces some phenomena, which are experimentally observed. Based on a VIV lift force model and the Morison equation, Liu et al. [6] developed a nonlinear time-dependent VIV model, which considered the fluid-structure interaction and took the in-line vibrations into account. The response-dependent lift force with nonlinear damping was considered to be one of the model characteristics, which make it different from other published ones. Their results showed that the presented model works well for describing the VIV of deepwater risers, because of its agreement with the published papers of the same study conditions using other models. The model was developed to simulate the cases under non-lock-in as a usual condition, under which the deep water risers are operated. Franzini et al. [7] introduced an experimental work of VIV with a rigid fixed flexible cylinder, and elastic mounted rigid cylinder as well, which was mounted on an elastic measurement device. Kwon et al. [8] investigated a drag reduction device that used three ribbons attached 120 degrees apart to vertical pipes to study the effects of the ribbon length and the direction of the flow on various current velocities. Their results presented that attached ribbons can be used to reduce the drag force on vertical pipes for various directions of incoming flows. Yang et al. [9] studied a three-dimensional nonlinear dynamic model of a long riser under vortex-induced vibration to determine the appropriate viscoelastic coefficients to suppress the nonlinear dynamic response. Their results demonstrated that the visco-elastic coefficients have a critical impact on the natural frequency of the riser. They also showed that appropriate visco-elastic coefficients are very important to effectively suppress the maximum displacements and stresses. Zhang et al. [10] studied experimentally and numerically for Reynolds number $30,000 \leq \mathrm{Re} \leq 110,000$. FIV of a single, rigid, circular cylinder with one-sided PTC (passive turbulence control). Comparisons of response are made between symmetric and one-sided PTC-cylinder. They showed that the asymmetrical oscillation and the effect of selective roughness are drawn with respect to the flow speed, damping ratios and the PTC locations angle at the end. 
The numerical solution of deep water risers using fluid-structure interaction approach had taken a considerable attention in the last years. Because of the high computing capacity needed to carry out such cases of simulation, some research papers went to simplify the regarded cases to be an elastic cantilever for the validation purposes. Di Silvio et al. [11] studied the fluid-elastic vibrations by using both mathematical modeling and experimental work. They had described the VIV as a physical phenomenon by using several mathematical methods. As a first step they had started to get the acting forces on the structure as a function of time. The vortex-origin time concept was regarded to determine the control volume on which the momentum equations will be built. The vortex-origin time is defined as the instant, in which the vortex is entering the stabilized wake. Then the fluid forces had been determined by assuming that the fluid domain is deforming in time as well as an influence of structural vibrations. It is clear that they had modeled an oscillated system. The experimental results had been analyzed and regarded to be a real application of the determined mathematical model. The experimental work had been done for both water and air as working fluids. That presented model was new at this time, because it regards the physical meaning of the wake width in controlling both the frequency and the amplitude of the alternate driving force. Tojo [12] tried to give more efficient fluid-structure interaction solver based on OpenFOAM standard solver ico-Fsi-Foam. The solver of him has actually main weak points, especially with handling the transient turbulent flows, since he had used SIMPLE algorithm to solve the fluid domain. As mentioned in the OpenFOAM user guide [13] the SIMPLE algorithm can handle the steady-state turbulent flows. The work of Habchi et al. [14] was very interesting and close to the present research paper. They presented a very robust solver for fluid-structure interaction based on OpenFOAM, which had been tested at Reynolds number of maximum 1000 which is below the natural conditions of risers environment. Yuan et al. [15] presented an alternative time domain force-decomposition model for flexible risers to predict VIV response under both steady and oscillatory flows. They showed that the VIV response under the oscillatory flows with high maximum decrement velocity needs to be studied with more related experimental facilities. Nguyen et al. [16] introduced numerically an investigation of wake-induced vibrations. Their numerical model depends on an incompressible Navier-Stokes flow solver with a hybrid detached eddy simulation approach for turbulence modelling. They found that Reynolds number has a critical impact in the response variation of amplitude and frequency of cylinders in wake-induced vibrations. Zhuang et al. [17] presented a modification of the shear-stress transport turbulence model to study the fluctuating lift and the 
drag of the cylinder in VIV. They utilized the embedded program of OpenFoam to perform the numerical modeling of VIV of a cylinder with two degrees of freedom.

In the present study the effect of three strakes shape in addition to the pitch of the helix by further examining in reducing the VIV of a flexible circular cantilever. Based on the literature reviews, the response of continuous helical strakes is still insufficient. Therefore, the novelty of the present work depends on non-continuous helical strakes in staggered arrangement to increase the turbulence intensity in the flow field around the risers. The proposed device was designed and numerically tested to achieve the following objectives;

1. Investigation the main deficiency and advantage of not-continuous helical strakes with three starts in reducing the VIV of a flexible cylinder.

2. Comparing the proposed design with continuous helical strakes and plain risers based on the drag force coefficient.

\section{Mathematical Modeling}

In this section, the used equation and mathematical interpretation for solving fluid and solid domains as well as coupling will be presented.

The fluid flow is governed by unsteady Navier-Stocks equations for viscous incompressible fluid. Since these equations are only valid for static meshes [18], the so-called Arbitrary LagrangianEulerian mapping must be used. This kind of mapping is very common to be used in fluidstructure interaction solvers.

The mapped governing equations could be written in the following form [19]:

$\nabla \cdot u_{f}=0$

$\frac{\partial u_{f}}{\partial t}+\left(u_{f}-u_{m, f}\right) \nabla u_{f}=-\frac{\nabla p}{\rho}+v_{f} \nabla^{2} u_{f}$

Where uf , um,f,$p$, of and $\rho$ f are fluid velocity in the static fluid domain at time zero $\Omega$ (f,0), mesh velocity in fluid domain $\Omega(\mathrm{f}, \mathrm{t})$, pressure and fluid density. The difference between fluid velocity and mesh velocity called the convective term.

A LES-one-equation model was used for the sub-gride scales (SGS), which solves one turbulent transport equation, usually the turbulent kinetic energy $k$. That eddy viscosity SGS model uses a modeled balance equation to simulate the behavior of $k$. 
The kinematic eddy viscosity could be represented as follows [20]:

$v_{T}=k^{\frac{1}{2}} l=C_{D} \frac{k^{2}}{\varepsilon}$

The used large eddy simulation model is given as follows [20]:

$\frac{d k}{d t}+U_{j} \frac{\partial k}{\partial x_{j}}=\tau_{i j} \frac{\partial U_{i}}{\partial x_{j}}-C_{D} \frac{k^{\frac{3}{2}}}{l}+\frac{\partial}{\partial x_{j}}\left[\left(v+\frac{v_{T}}{\sigma_{k}}\right) \frac{\partial k}{\partial x_{j}}\right]$

The solid domain had been solved by using the incremental strain updated Lagrangian approach. This mathematical modeling allows strains in a good range, which is needed in the field of deep risers engineering as well as the validation study case of the present paper. This part of solver had been built as explained in the research paper of Tuković et al. [19]. The motion of an iso-thermal continuum in an arbitrary volume $\mathrm{V}$ bounded by a surface $\mathrm{S}$ is governed by the conservation laws for mass and linear momentum. The final formulation of the momentum equation, which is ready for discretization, is given as follows [21]:

$\int_{V_{u}} \rho_{u} \frac{\partial \delta \mathrm{v}}{\partial t} \mathrm{~d} V_{u}-\oint_{S_{u}} \mathrm{n}_{u} \cdot(2 \mu+\lambda) \nabla \delta \mathrm{u} \mathrm{d} S_{u}=\oint_{S_{u}} \mathrm{n}_{u} \cdot \mathbf{q} \mathrm{d} S_{u}$

where $\rho, \mathrm{n}, \mathrm{v}, \mathrm{Vs}, \sigma$ and $\mathrm{fb}$ are the continuum density, the outward pointing unit normal to the surface $S$, the velocity of the continuum, the velocity of the surface $S$, the Cauchy stress tensor and the resulting body force respectively.

Adaptive iterative under-relaxation and a strong coupling algorithm Aitken had been used. The coupling formulation could be written as follows [21]:

$\delta \tilde{\mathrm{u}}_{I, k}=\omega_{k} \delta \mathrm{u}_{I, k}+\left(1-\omega_{k}\right) \delta \tilde{\mathrm{u}}_{I, k-1}$

$\omega_{k}=1-\gamma_{k}$

$\gamma_{k}=\gamma_{k-1}+\left(\gamma_{k-1}-1\right) \frac{\left(\Delta \delta \mathrm{u}_{k-1}-\Delta \delta \mathrm{u}_{k}\right) \cdot \Delta \delta \mathrm{u}_{k}}{\left(\Delta \delta \mathrm{u}_{k-1}-\Delta \delta \mathrm{u}_{k}\right)^{2}}$

Where: $\Delta \delta \mathrm{u}_{k}=\delta \tilde{\mathrm{u}}_{k-1}-\delta \mathrm{u}_{k}$ and $\Delta \delta \mathrm{u}_{k-1}=\delta \tilde{\mathrm{u}}_{k-2}-\delta \mathrm{u}_{k-1}$

This mathematical modeling had been processed based on OpenFOAM 3.0-ext [15], which namely is an open source $\mathrm{C}++$ toolbox for computational mechanics. The tools of this release of OpenFOAM had been used to approximate the fluid flow and structure displacement by using the 
finite volume discretization. Moreover, a linear interpolation scheme had been used to interpolate the cell data to face data.

The velocity-based method of Laplacian smoothing had been used for moving the mesh. This part of solver had been built based on the formulation of Laplacian smoothing in both of papers Tuković et al. [21] and Lohner et al. [22].

\section{Solver Algorithms}

This section will explain the main parts of the FSI solver, by which the cases in the present study were numerically investigated. The PIMPLE algorithm is used for coupling the pressure and velocity fields. The PIMPLE solving algorithm is a hybrid of PISO (Pressure Implicit with Splitting of Operators) and SIMPLE (Semi-Implicit Method for Pressure-Linked Equations) algorithms. The PIMPLE main structure is inherited from the original PISO, but it allows equation underrelaxation, as in SIMPLE, to ensure the convergence of all the equations at each time. Figure (1) has a flow diagram showing the procedure of the PIMPLE loop, which is implemented in the solver. For stable convergence, adaptive time step and precise results, the Courant number was fixed at 0.7 in all simulations (except for the cavity validation case). For the cavity validation case, a time step dependency test was carried out and therefore the time step was fixed. By using a finite volume method, the solution of the solid domain is determined. The solid solver has to obtain both solid domain deformation and velocities. The main file of solid solver will initially check the closed temporal derivation scheme. The solid solver algorithm then calls either the Euler or backward scheme solver. Both solver algorithms are typically the same, but they differ in the way they deal with the results of the previous time-iteration. Figure (2) shows the flow diagram of the main header algorithm of the solid solver. The visualization of the flow in the wake of the cylinder had been discussed and analyzed for large-eddy simulation (LES), SpalartAllmaras - SA and Spalart Allmaras improved delayed detached eddy simulation - S-A IDDES turbulence models. The results showed that the LES is highly recommended for solving the flow systems of high reynolds number cross flow around a circular cylinder such as deep water risers' cases under OpenFOAM. Figure (3) presents a comparison of time-averaged drag coefficient with different Reynolds number of published numerical and experimental results versus the present work for plain cylinder. This figure shows that, unlike LES, both SA-IDDES and SA showed bad agreement with the published numerical and experimental results. The $y+$ value for all cases, in which turbulence takes place, is 0.8 . 


\section{Code Validation}

This section discusses the numerical results when compared with the published experimental and numerical benchmarks. The validation cases were simulated under the environmental conditions to give a reasonable and fair comparison. The validation had been carried out using an experimental benchmark of a flexible vertical cylindrical cantilever shown in Figure (4). The values and geometry have been determined depending on the experiment of Franzini [7]. Two values of reduced velocity had been considered for validation. The reduced velocities of 3.69 and $6.03 \mathrm{had}$ been selected for the mentioned benchmark case experiments. As mentioned, the results are compared to the experimental work of Franzini [7] for the aim of validation. Table (1) shows that comparison. As presented in Table (2), the relative variation between the present numerical model and previously experimental results for $\mathrm{x} / \mathrm{D}$ and $\mathrm{y} / \mathrm{D}$ vary between $3.8 \% 19.4 \%$. This variation may be because VIV is a complex fluid-structure interaction behavior, it has lots of factors involved in this process and thus quite difficult to predict high accuracy results in most simulation models. Also, the CFD results depend to the turbulence model. Although LES produced good agreement with the published numerical and experimental results as shown in Figure (3) than other models, it did not capture the maximum dimensionless displacement of the midpoint on the cantilever free end in $\mathrm{y}$-direction $\mathrm{y} / \mathrm{D}$ well. The reduced velocity is expressed as $u^{*}=U / f_{1} D$, where $U$ is the flow velocity at the inlet, $f_{1}$ is the first natural frequency of the cantilever and $D$ is the cantilever diameter.

\section{Numerical Domain Setup}

\subsection{Problem statement}

The considered case of a straked flexible cantilever subjected to a water cross flow has the same design and operational parameters as the validation case of a bar cantilever. The only difference between both designs is adding the strakes as a suppression device to the cantilever. The straked cantilever has a free and a fixed end as well. The concept of the mentioned device depends on attaching vortex generators on the both sides of the riser. The vortex generators are placed where the separation points are relocating. Adding such vortex generators are aiming to prevent the continues relocation of the flow separation points and hence the vortex shedding. The geometry of the cross sectional shape of the suppression devices are shown in Figures (5) and (6) (all dimensions in $\mathrm{mm}$ ), which will be called "shape two", while "shape one" is a straked cylinder 
with three starts and continuous helical restricting. The shape two is a cylinder with noncontinuous helical strakes in staggered arrangement with two starts. The strake shape designs are presented in Table (3). Figure (7) shows the geometry of the considered computational domain with boundary conditions. The upper and lower boundaries in z-direction, namely perpendicular to the Figure (7), are considered to be symmetry planes, while the zero-velocity boundary condition is considered for "walls" surfaces. The fluid is pure water with a density of $1000 \mathrm{~kg} / \mathrm{m} 3$ and a kinematic viscosity of $10-6 \mathrm{~m} 2 / \mathrm{s}$ flows at $0.34 \mathrm{~m} / \mathrm{s}$, which results in a flow of Reynolds number $10_{4}$ around the elastic circular cantilever. The elastic cantilever is made from Polyvinyl Chloride of Young's modulus $1.5 \times 109$ Pascal and density $1500 \mathrm{~kg} / \mathrm{m} 3$ as well as a Poisson's ratio of 0.4. The main dimensionless parameters of design and material are listed in Table (4).

Where $S, D, l, m *, f 1$ and EI are the distance between cantilever moving end and the bed of the water tank, the cantilever diameter, the cantilever length, the mass ratio $m *=$ the mass of the oscillating body divided by the mass of displaced fluid, the first natural frequency and the bending stiffness respectively.

\subsection{Mesh and discretization}

A fluid domain has been divided into 641598 tetrahedral cells. The mesh near to the cylinder wall is refined for better wall treatment, at which the interaction between both domains are taking place. The mesh refinement at the interaction region aims to insure high accuracy at forces transfer, which is playing the main role in the vibrations of the elastic cylinder. The concept of meshing was to focus on the accuracy of vibration rather than downstream flow, since the save of computational resources was important. Figure (8) shows the mesh used in the staked cylinder with pitch $=15 \mathrm{D}$ simulation in different cross sectional views. A mesh independence test has been carried out to ensure the accuracy of results in terms of discretization error for each case. Figures (9-11) show the mesh independence tests for the shape two, shape one and plain cylinder respectively. These mesh sizes give a small change in results after more refinement.

\section{Results discussion and analysis}

\subsection{Effect of helical strakes shape}

\subsubsection{Plain cylinder}

Figure (12) shows three-dimensional contours of pressure in wake zone for plain cylinder. The contours results at different time steps show the vortex shedding behind the elastic cantilever 
and colored by velocity values. Moreover, Figure (13) shows the two-dimensional vorticity contours at $\mathrm{z}=0.2$ meters from the bottom wall. The results gave a maximum drag coefficient of 1.51 .

\subsubsection{Straked shape one}

In Figure (14), the three dimensional pressure contours of the flow around the cantilever at different times are presented and colored with velocity values. The mentioned three dimensional pressure contours give a good visualization of the wake zone in down stream. Figure (15) shows the vorticity contours at different times. The vorticity around z-direction is considered, as it represents the more important parameter regarding the topic of research and to give the chance for fair comparison. By comparison, between the results in Figure (15) and results for plain cylindrical cantilever, we show that the vortex-shedding has been developed in a clear form behind the cylinder, which means that the strakes on the surface of the cantilever has successfully minimized the formation of vortices in down stream. Also, by visually comparing among the results in Figure (15) and results for plain cylindrical cantilever, it would be observed the clear understanding of the difference between both geometries in terms of vortex shedding in down stream. The results gave a maximum drag coefficient of 1.3. The reason for which the drag coefficient is clearly reduced by adding on the strakes is increasing the turbulence intensity in the flow field around the cylindrical cantilever.

The straked cantilever showed a reduced $y / D$ value. By comparing the maximum $y / D$ calculated values of both cases, it is observed that the straked shape has reduced the crossdirectional vibrations by 73 percent. The plain and straked shapes gave a y/D value of 0.76 and 0.21 respectively.

\subsubsection{Straked shape two}

In the present section, a straked shape one is modified as shown in Figure (6) which illustrates the geometry of the modified shape (shape two) of the suppression device. The concept of the proposed modification depends on attaching vortex generators on the both sides of the riser, this vortex generator is non-continuous helical strakes in staggered arrangement. The vortex generators are placed where the separation points are relocating. Figures (16) and (17) show the velocity and vorticity contours are given at different sections and time steps of the straked shape two. The two mentioned figures give the chance of understanding the flow in down stream behind the proposed suppression device, in terms of visual observation of the eddies 
formation in downstream. As shown Figures (16) and (17), the formation of vortex shedding has been weakened as a result of adding the side vortex generators. Adding such vortex generators are aiming to prevent the continues relocation of the flow separation points and hence the vortex shedding. Figure (18) shows the time history of both lift and drag coefficients. The results gave a maximum drag coefficient of 1.05 is considered reduced value if compared to the value of the straked cylinder with shape one. Also, this figure shows the maximum value of lift coefficient is about 0.45 .

The drag and lift coefficients have been calculated as:

$$
\begin{aligned}
& C_{D}=\frac{2 F_{D}}{A \rho_{\infty} U^{2}} \\
& C_{L}=\frac{2 F_{L}}{S \rho_{\infty} U^{2}}
\end{aligned}
$$

where $F_{L}$ is the lift force, $F_{D}$ is the drag force, $A$ is the reference area of the obstetrical, and $S$ is the relevant plan area of the obstetrical.

The suppression efficiency $S E$ can be obtained by using the Eq. (11):

$S E=\frac{\left(\frac{y}{D}\right)_{\text {Franzini }}-\left(\frac{y}{D}\right)_{\text {Shape }} \text { wo }}{\left(\frac{y}{D}\right)_{\text {Franzini }}} \times 100$

According to the values of $y / D$ in Lissajous curve as shown in Figure (19), the suppression efficiency of the modified strakes shape two was $88.72 \%$. The value of $y / D$ for bar obstacle has been taken from the experimental work of Franzini [7].

\subsection{Effect of helical strakes pitch}

In the present section, a straked shape one of a rigid continuous helical obstacle (shape one) is considered. The selected design parameter is the pitch of the helical shape of strakes. The pitch of strakes varied between 1D to 17D with an increment of 2D. Figures (20) and (21) give a visual representation of the flow in downstream. Figure (20) shows the velocity contours at different pitch values. The results were taken at the time step, which represents the steady state of wake flow behind the obstacle. Figure (21). shows the vorticity around y-direction, which is the perpendicular direction to the figure. The shown contours were taken at the time step, which represents the steady state of wake flow behind the obstacle. The results give a good visual representation to the vortex shedding of downstream flow. Also, Figure (22) shows the change of 
the both times averaged drag and lift coefficients with respect to pitch of strakes. The results show that increasing the pitch value has reduced lift and increased drag force coefficients.

Table (5) presents a conclusion for the results comparison among the three designs; plain cylinder, stroke one and struck two.

\section{Conclusions}

This paper presents a numerical simulation investigation to study the effect of new suppression device attached to a deep water risers subjected to a cross water flow at $\operatorname{Re}=104$. The VIV effect with regard to different geometries and design parameters such as the effect of the pitch and shape of strakes are studied. The presented design has been tested using an OpenFOAM fluid-structure interaction solver, which had been presented and validated. From the present results the following points were concluded:

1. Numerical solution for suppression device of vortex-induced vibrations in deep water risers have been introduced and numerically investigated.

2. A numerical solver based on OpenFoam $\mathrm{C}++$ platform concerned with fluid-structure interaction problems is presented.

3. The solver validation had been done by comparing its results with published experimental and numerical results and had shown a good agreement.

4. The drag coefficient of 1.05 is considered a reduced value in the modified strakes shape two.

5. The formation of vortex shedding has been weakened as a result of adding the side vortex generators according to the modified strakes shape to design and achieved a suppression efficiency about $88.72 \%$ compared to the plain cantilever.

\section{NOMENCLATURES}

\section{Latin Symbols}
$A$ area, $\mathrm{m}_{2}$
$y \quad$ vibration amplitude in $\mathrm{X}$ direction, $\mathrm{m}$.
$x \quad$ vibration amplitude in $\mathrm{Y}$ direction, $\mathrm{m}$.
D cylinder diameter, $m$. 
e gap, m.

$k$ turbulent kinetic energy, $\mathrm{J} / \mathrm{kg}$.

$u \quad$ velocity vector, $\mathrm{m} / \mathrm{s}$

$U$ velocity, $\mathrm{m} / \mathrm{s}$

$p$ pressure, $\mathrm{N} / \mathrm{m} 2$

t time, sec.

CD drag coefficient, dimensionless.

$\mathrm{CL}_{\mathrm{L}}$ lift coefficient, dimensionless.

q energy, J/kag.

$f$ natural frequency, s-1.

$F$ force, N.

$l \quad$ length, $\mathrm{m}$.

$S$ distance, $\mathrm{m}$.

$m *$ mass ratio, dimensionless.

$v *$ velocity ratio, dimensionless.

$S E$ suppression efficiency, $\%$.

EI bending stiffness, N.m2.

\section{Symbols}

$v$ kinetic viscosity, $\mathrm{m}_{2} / \mathrm{s}$

$\rho$ density, $\mathrm{kg} / \mathrm{m} 3$

$\mu$ dynamic viscosity, $\mathrm{kg} / \mathrm{m} . \mathrm{s}$

\section{Subscripts}
m mesh
f fluid
1 first
$\mathrm{S}$ surface

\section{Abbreviation}


CFD Computational Fluid Dynamics

FSI Fluid-Structure Interaction

FIV Flow-Induced Vibrations

LES Large Eddy Simulation

OF OpenFOAM open source software

Re Reynolds Number

VIV Vortex-Induced Vibrations

PISO Pressure Implicit with Splitting of Operators

SIMPLE Semi-Implicit Method for Pressure-Linked Equations

PTC Passive Turbulence Control

SGS Sub-Gride Scales

SE Suppression Efficiency 


\section{Ethical Statement for International Journal of Nonlinear \\ Dynamics and Chaos in Engineering Systems}

I testify on behalf of all co-authors that our article submitted to the International Journal of Nonlinear Dynamics and Chaos in Engineering Systems:

Title: Numerical Study of New Device for Passive Suppression of Vortex-Induced Vibrations in Deep Water Risers

All authors: Prof. Dr. Mohammed Abdulaziz

\section{Prof. Dr. Emad M.S. El-Said}

1) The manuscript submitted represents our own original work and has not been published and is not being submitted or considered for publication elsewhere;

2) All authors have been personally and actively involved in substantive work leading to the manuscript, and will hold themselves jointly and individually responsible for its content.

3) All authors have contributed to the manuscript and that common agreement has been reached before submission.

4) All authors participated in the work in a substantive way and are prepared to take public responsibility for the work.

5) The text, illustrations, and any other materials included in the manuscript do not infringe upon any existing copyright or other rights of anyone.

6) The authors are agreeing that all materials submitted for evaluation (including text, figures, etc.) retains the property / copyrights of the International Journal of Nonlinear Dynamics and Chaos in Engineering Systems. In the case of not acceptable papers, after their rejection the property of all materials will backward to the authors.

7) Reproduction, posting, transmission or other distribution or use of the article or any material contained therein, requires a citation to the International Journal of Nonlinear Dynamics and Chaos in Engineering Systems.

Date: 27-04-2019

Corresponding author's signature:

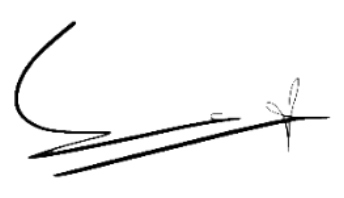




\section{Acknowledgements}

Mohammed Abdulaziz thanks the guidance from Professor Bettar Ould ELmoctar ( University of Duisburg Essen ) and Professor Wojciech Kowalczyk ( University of Duisburg Essen ).

\section{Authors' contributions}

Conceptualization, MA; methodology, MA and EE; software, MA; validation, MA; writingoriginal draft preparation, EE; writing-review and editing, MA. All authors read and approved the final manuscript.

\section{Funding}

Not applicable.

\section{Availability of data and materials}

Materials described in the manuscript, including all relevant raw data, will be freely available to any scientist wishing to use them for non-commercial purposes upon request via e-mail with corresponding author.

\section{Ethics approval and consent to participate}

Not applicable.

\section{Consent for publication}

Not applicable.

\section{Competing interests}

The authors declare no competing interests 


\section{References}

[1] Jeans, G., Prevosto, M., Harrington-Missin, L., Maisondieu, C., Herry,C., Lima, J.,2012. deep water current profile data source for riser engineering offshore. Brazil, ASME 31 st International Conference on Ocean, Offshore and Arctic Engineering.

[2] Theodore von Kármán, Aerodynamic, McGraw-Hill, 1963.

[3] Kim, S., Lee,S.C., 2012. Suppression of flow-induced vibration of a circular cylinder by means of a flexible sheet. Journal of Mechanical Science and Technology 26 (6), 1773-1779.

[4] Li, X., Wang, G., Wang,Y., Jiang, M., He, X., 2013. Identification of Hydrodynamic Forces on a Flexible Pipe Near Plane Boundary Subjected to Vortex-Induced Vibrations. J. Shanghai Jiaotong Univ. (Sci.) 18 (1), 44-53.

[5] Xu,W.H., Gao, X.F., Du, J., 2012. The prediction on in-line vortex-induced vibration of slender marine structures. Acta Mechanica Sinica, 28 (5) 1303-1308.

[6] Liu, J., Huang, W., 2013. A Nonlinear Vortex Induced Vibration Model of Marine Risers. Journal of ocean university of China 12, 32-36.

[7] Franzini, G.R., Pesce1, C.P., Gonçalves, R.T., Fujarra, A. L.C., Pereira, A.P., 2011. Concomitant Vortex Induced Vibration Experiments: a Cantilevered Flexible Cylinder and a Rigid Cylinder Mounted on a Leaf-spring Apparatus. Proceedings of the XIV International Symposium on Dynamic Problems of Mechanics, Brazil.

[8] Kwon,S., Cho, J., Park, J., Choi, H., 2002. The effects of drag reduction by ribbons attached to cylindrical pipes. Ocean Engineering, 29, 1945-1958.

[9] Yang,W., Ai, Z., Zhang, X., Gou, R., Chang, X., 2018. Nonlinear three-dimensional dynamics of a marine viscoelastic riser subjected to uniform flow, Ocean Engineering 149, 38-52.

[10] Zhang, D., Sun, H., Wang, W., Bernitsas, M.M., 2018. Rigid cylinder with asymmetric roughness in Flow Induced Vibrations. Ocean Engineering 15, 363-376.

[11] Di Silvio, G., Angrilli, F., Zanardo, A., 1975. Fluidelastic viberations: mathematical model and experimental results. Meccanica. 
[12] Tojo, B.M., 2012. Aero-Structural Blade Design of a High-Power Wind Turbine, Instituto Superior T’ecnico, Technical University of Lisbon, Portugal.

[13] http://www.openfoam.org/features/standard-solvers.php (accessed on 01.07.2014)

[14] Habchi, C., Russeil, S., Bougeard, D., Harion, J., Lemenand, T., Ghanem, A., Valle, D., Peerhossaini, H., 2013. Partitioned solver for strongly coupled fluid-structure interaction. Computers \& Fluids. 71, 306-319.

[15] Yuan Y., Xue H., Tang W., 2018. Numerical analysis of Vortex-Induced Vibration for flexible risers under steady and oscillatory flows, Ocean Engineering, 148, 548-562.

[16] Nguyen V., Chan W.H.R., Hoang H.N., 2018. Numerical investigation of wake induced vibrations of cylinders in tandem arrangement at subcritical Reynolds numbers, Ocean Engineering, 154, 341-356.

[17] Zhuang K., Zhang C., Ma G., Ni W., 2018. A numerical investigation of two-degree-offreedom VIV of a circular cylinder using the modified turbulence model, Ocean Engineering, $155,211-226$.

[18] http://www.extend-project.de (accessed on 01.07.2014)

[19] Tuković, Ž., Jasak, H., 2007. Updated Lagrangian Finite Volume Solver for Large Deformation Dynamic Response of Elastic Body, Transactions of FAMENA, 31 (1), 55-70.

[20] Sagaut P., 2006. Large Eddy Simulation for Incompressible Flows, Third Edition, Springer.

[21] Tuković, Ž., Jasak, H., 2004. Automatic Mesh Motion for the Unstructured Finite Volume Method, Elsevier Science.

[22] Lohner, R., Yang, C., 1996. Improved ale mesh velocities for moving bodie. Commun Numer Methods Eng., 12 (10), 599-608.

[23] Pontaza, P., Chen, H., Chen, C., 2006. final project report: CFD Simulation of Riser VIV, MMS Project Number 481. 
[24] Kassiotis, C., Ibrahimbegovic, A., Niekamp, R., Matthies, H., 2011. Nonlinear fluid structure interaction problem. Part I: implicit partitioned algorithm, nonlinear stability proof and validation examples, Comput Mech, 47, 305-23.

[25] Vazquez, J.G.V., 2007. Nonlinear analysis of orthotropic membrane and shell structures including fluid-structure interaction, Ph.D. thesis, Escola Tecnica Superior d'Enginyers de Camins, Universitat Politecnica de Catalunya, Barcelone, Espagne.

[26] Mok, D.P., 2001. Partitionierte losungsansatze in der strukturdynamik und der fluidstrukturinteraktion, Ph.D. thesis, Universitat Stuttgart, Holzgartenstr. 16, 70174 Stuttgart.

[27] Gerbeau, J.F., Vidrascu M., Frey P., 2005. Fluid-structure interaction in blood flows on geometries based on medical imaging. Comput Struct, 83(2-3), 155-65.

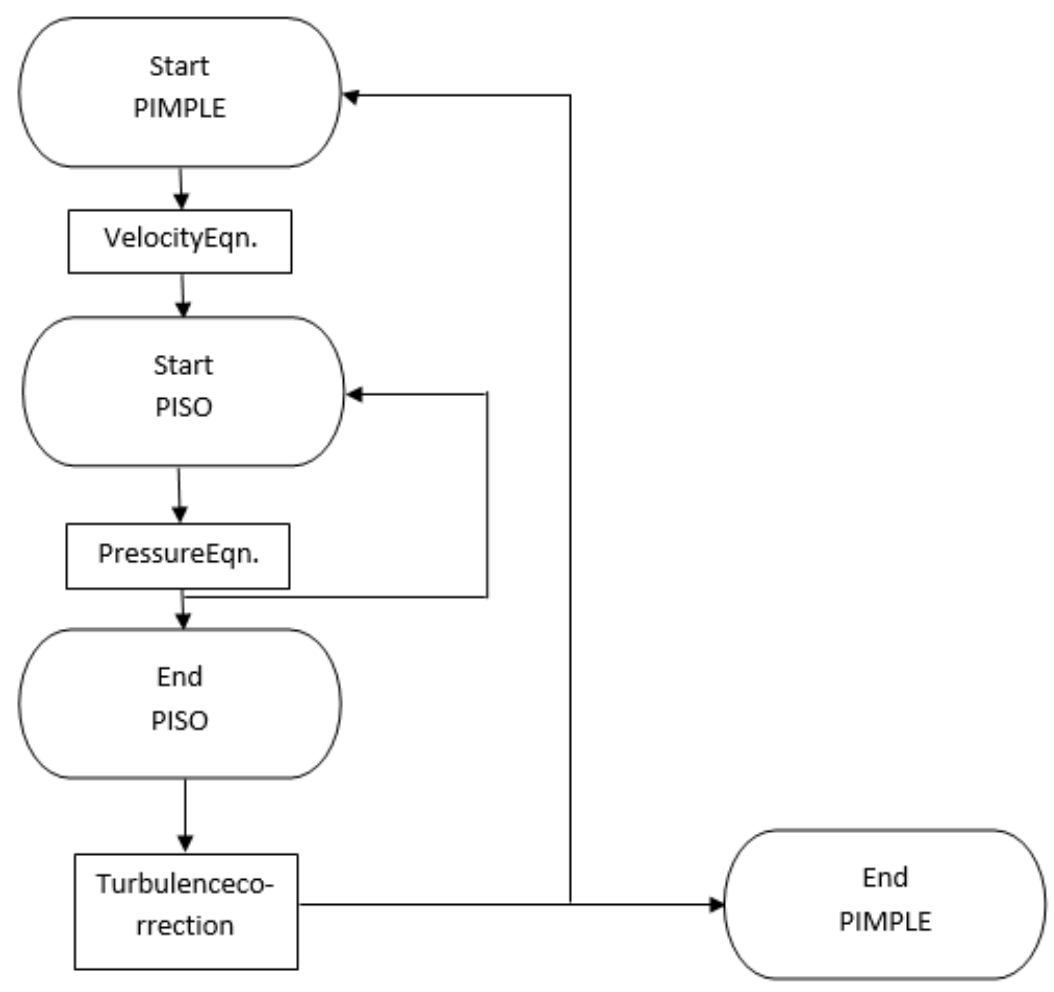

Fig. 1. PIMPLE algorithm flow diagram. 


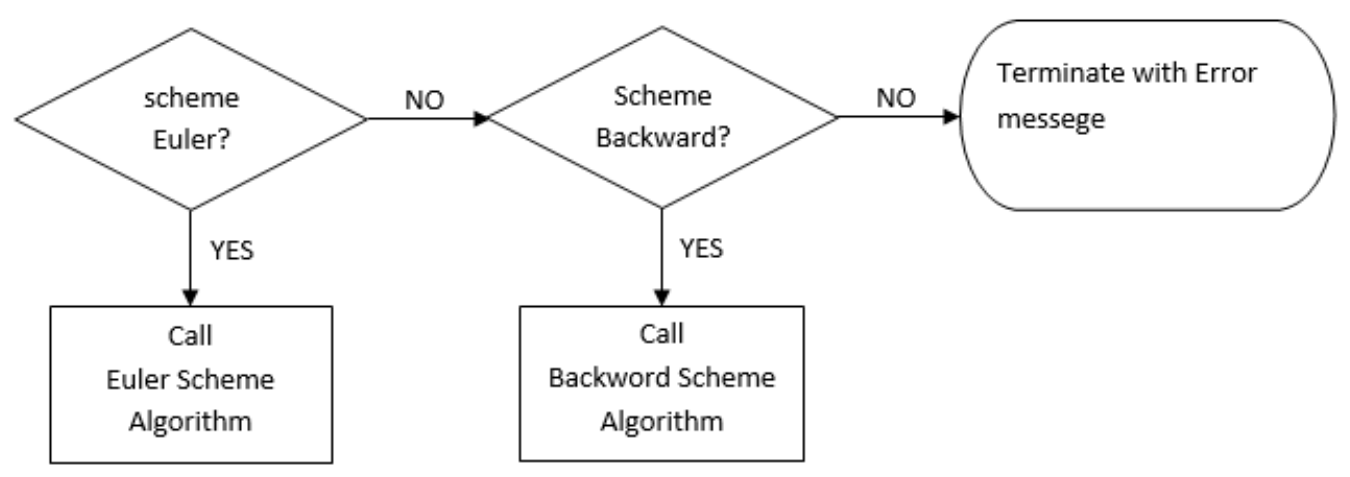

Fig. 2. Solid solver algorithm flow diagram.

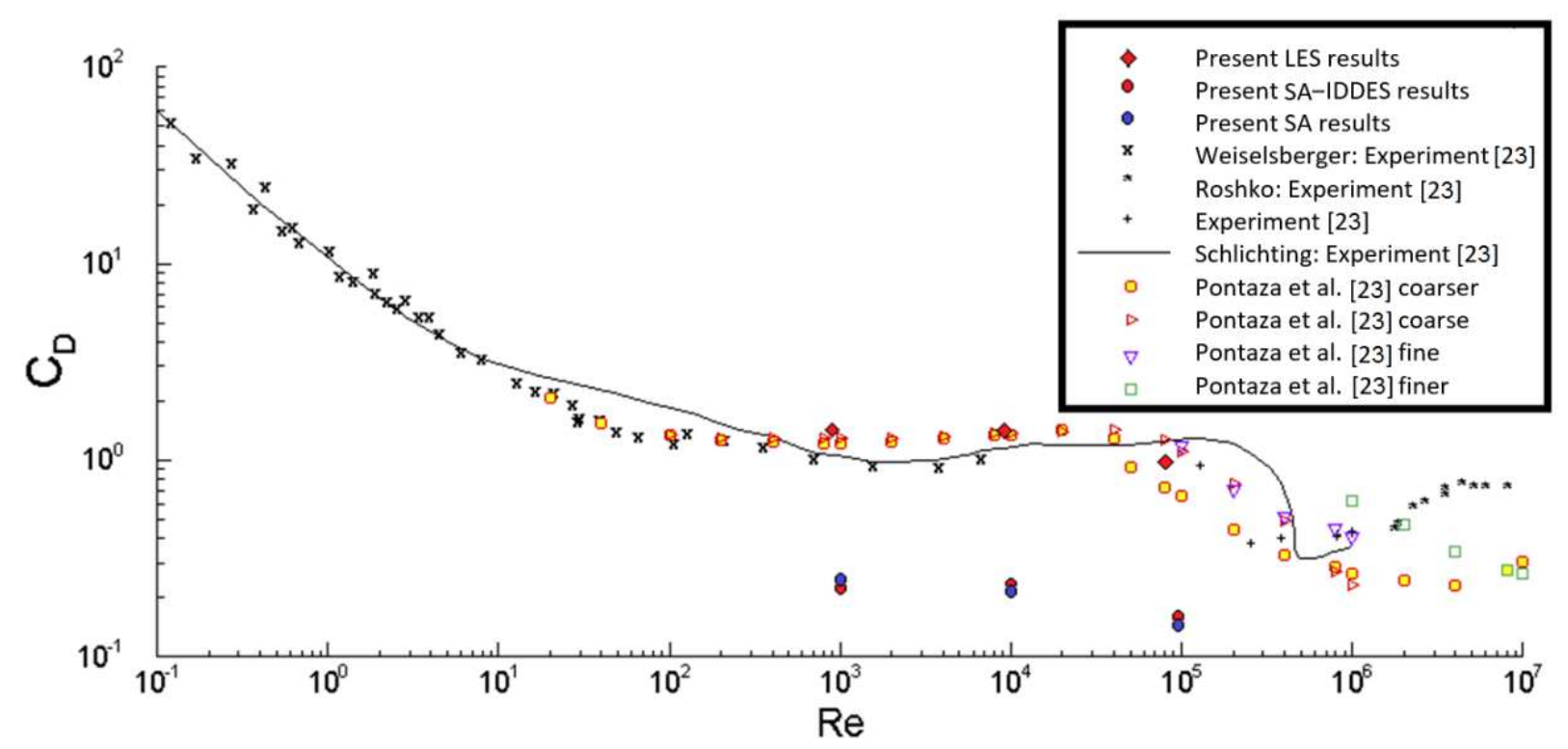

Fig. 3. Time-averaged drag coefficient with different Reynolds number of the published numerical and experimental results by [23] compared with the present results. 


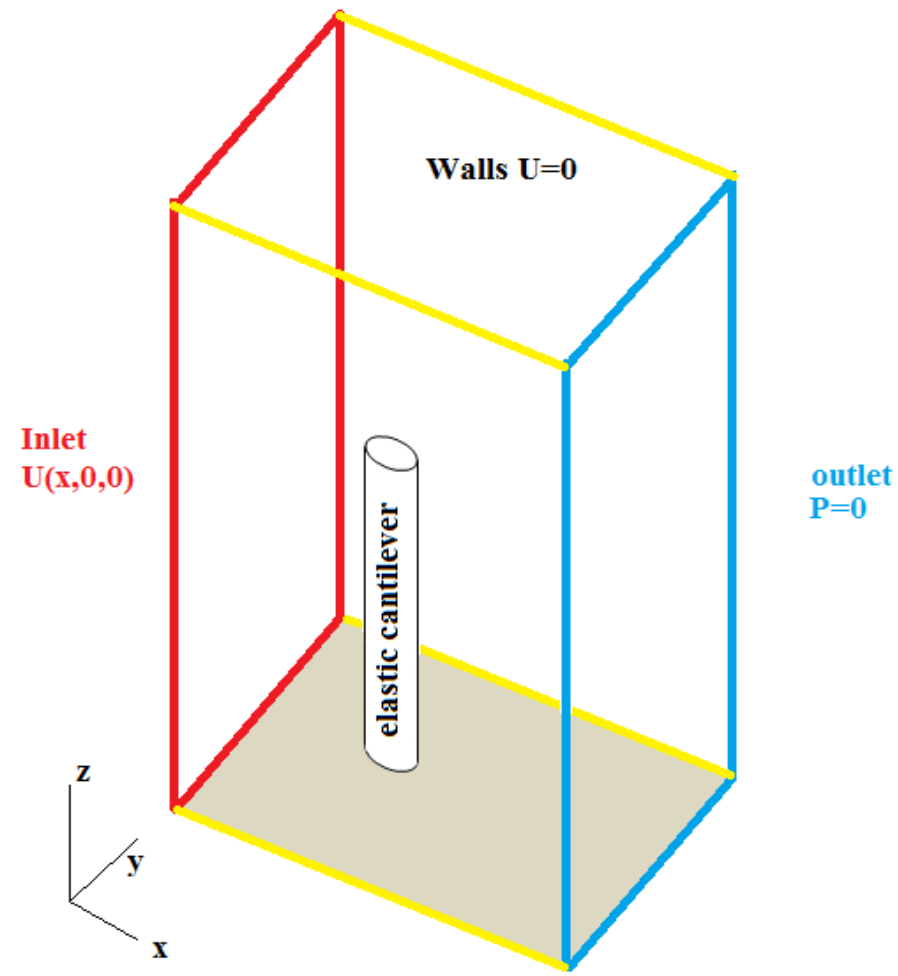

Fig. 4. An isometric view of the elastic cantilever study case, showing both fluid and solid domains as well as considered boundary conditions.

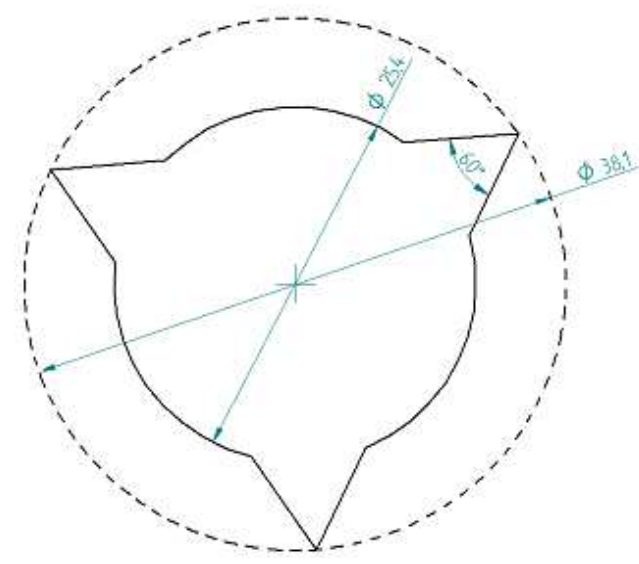

Fig. 5. The geometry and design parameter of of the suppression device; strake shape one. 

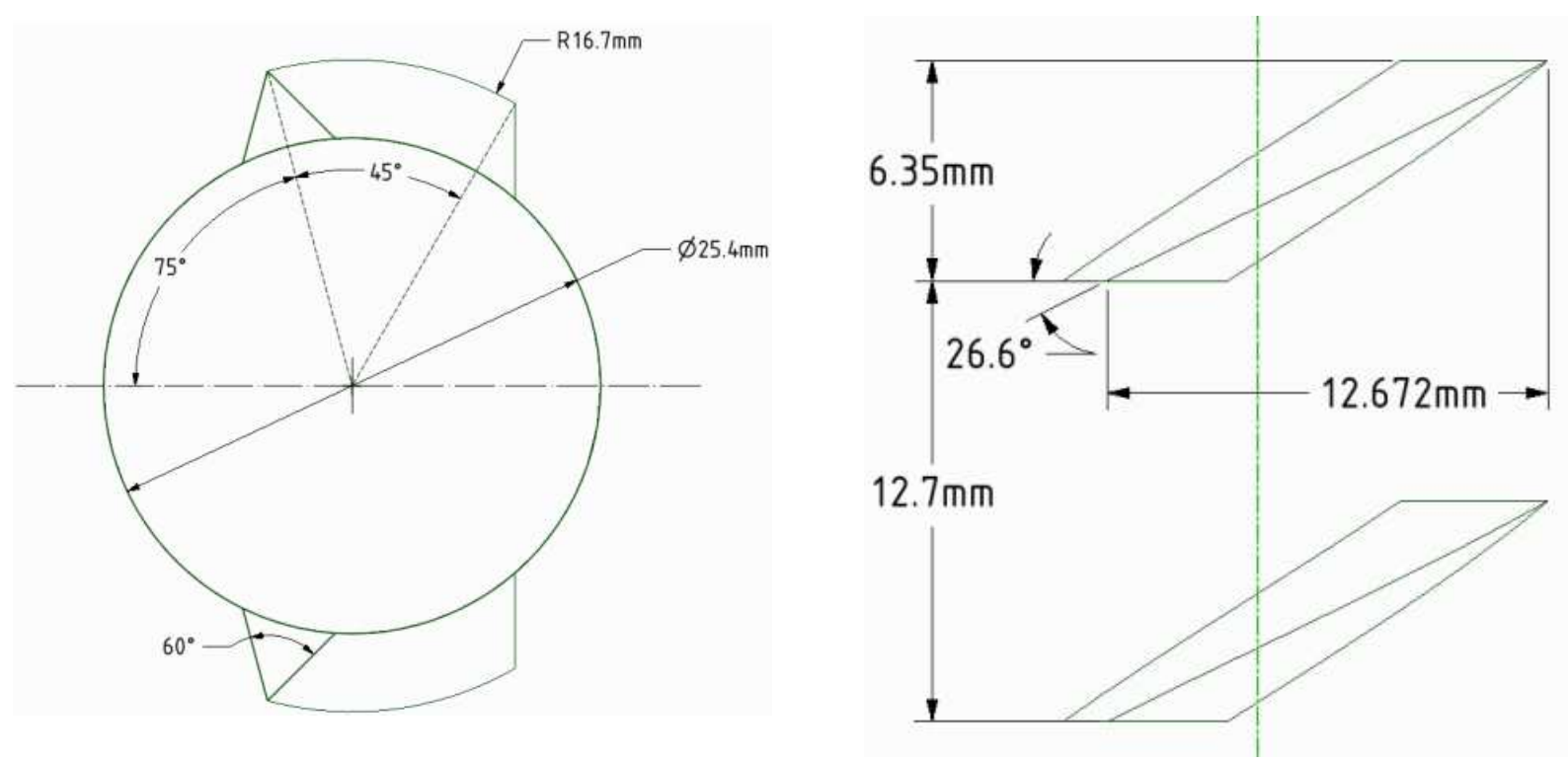

Fig. 6. The geometry and design parameter of the suppression device; strake shape two.

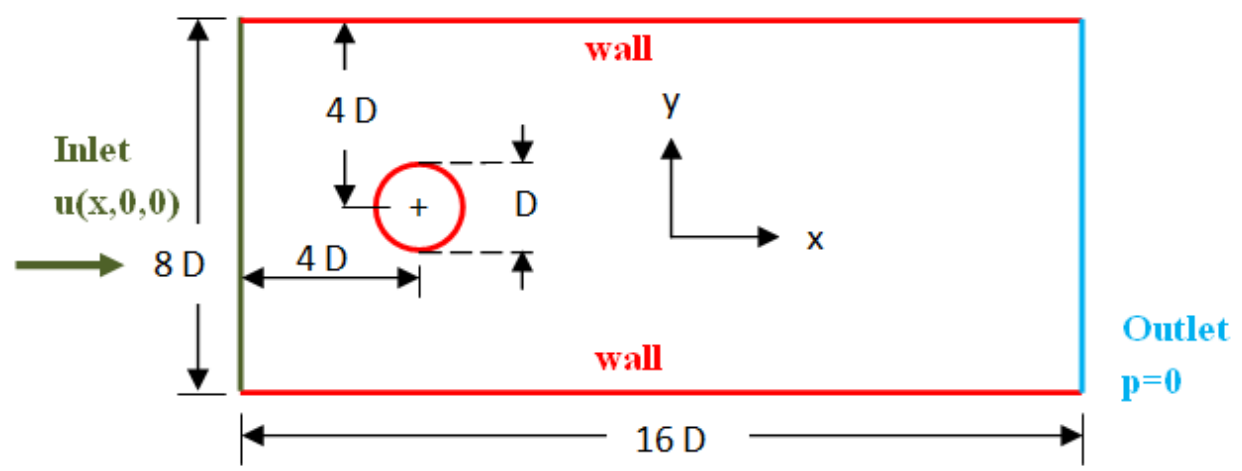

Fig. 7. A geometrical view of the stationary cylinder case showing the boundary conditions.

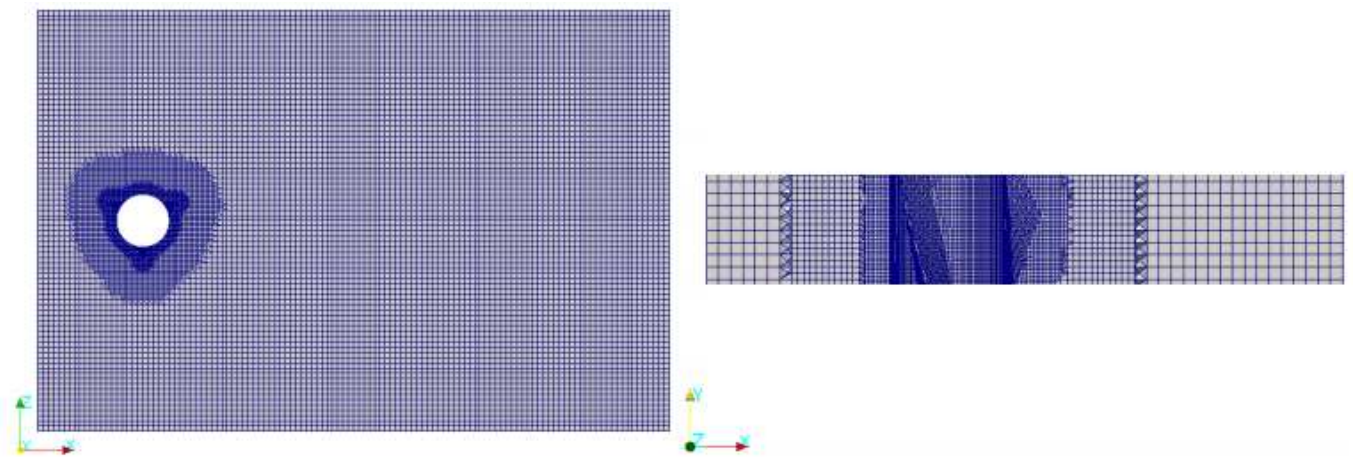

Fig. 8. Mesh of the staked cylinder with pitch $=15 \mathrm{D}$. 


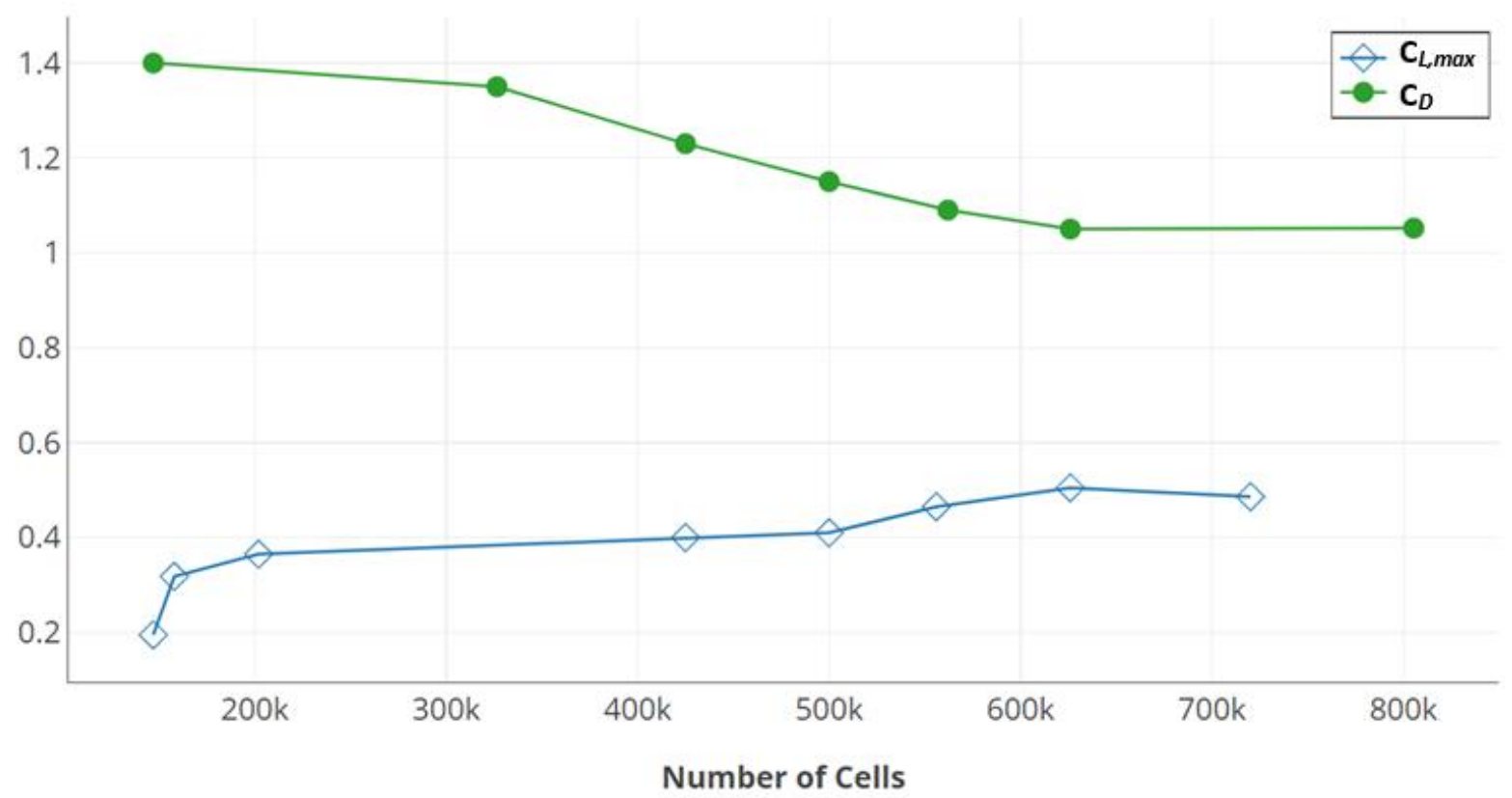

Fig. 9. Mesh dependency test results for the shape two.

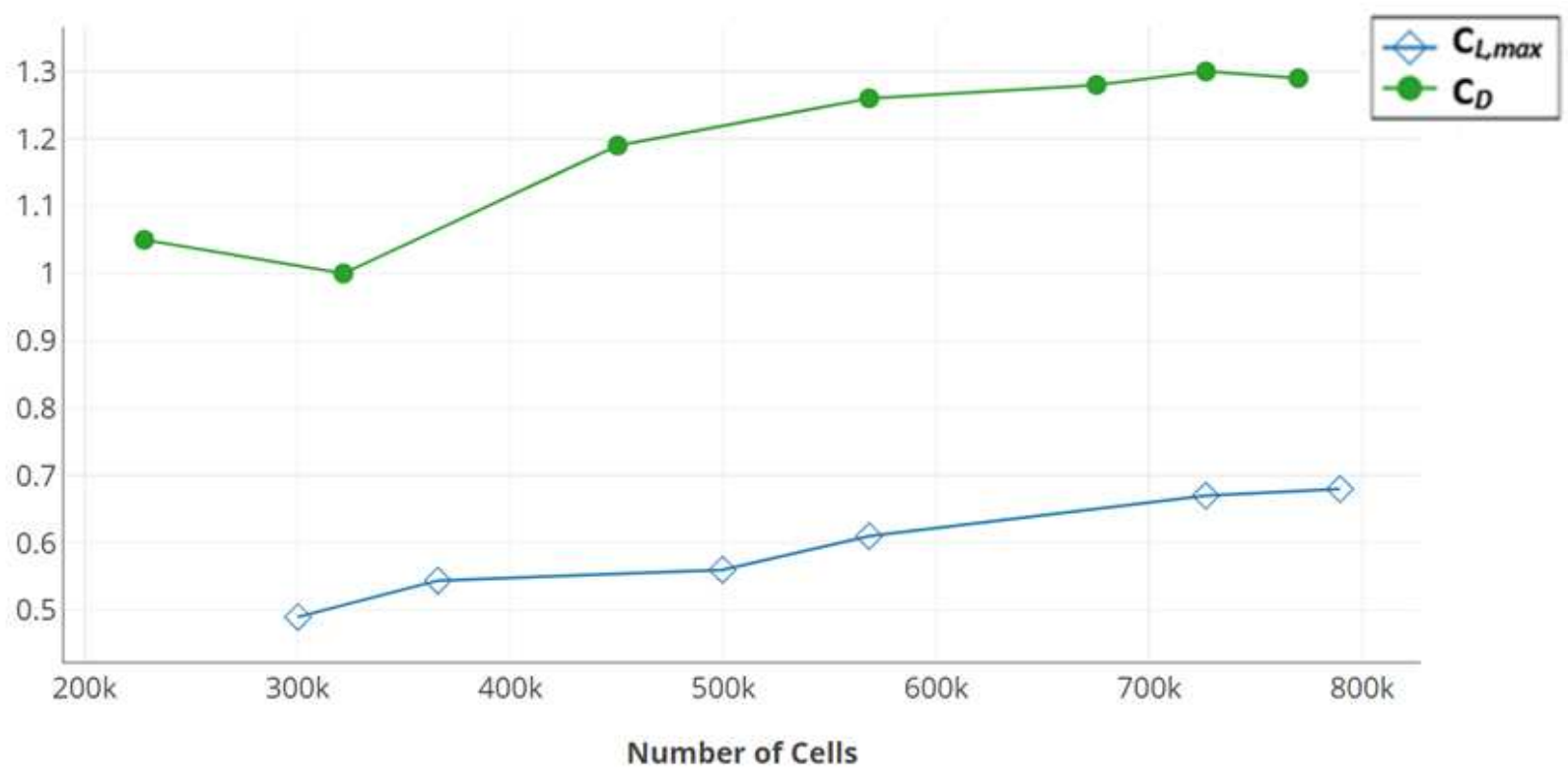

Fig. 10. Mesh dependency test results for the shape one case. 


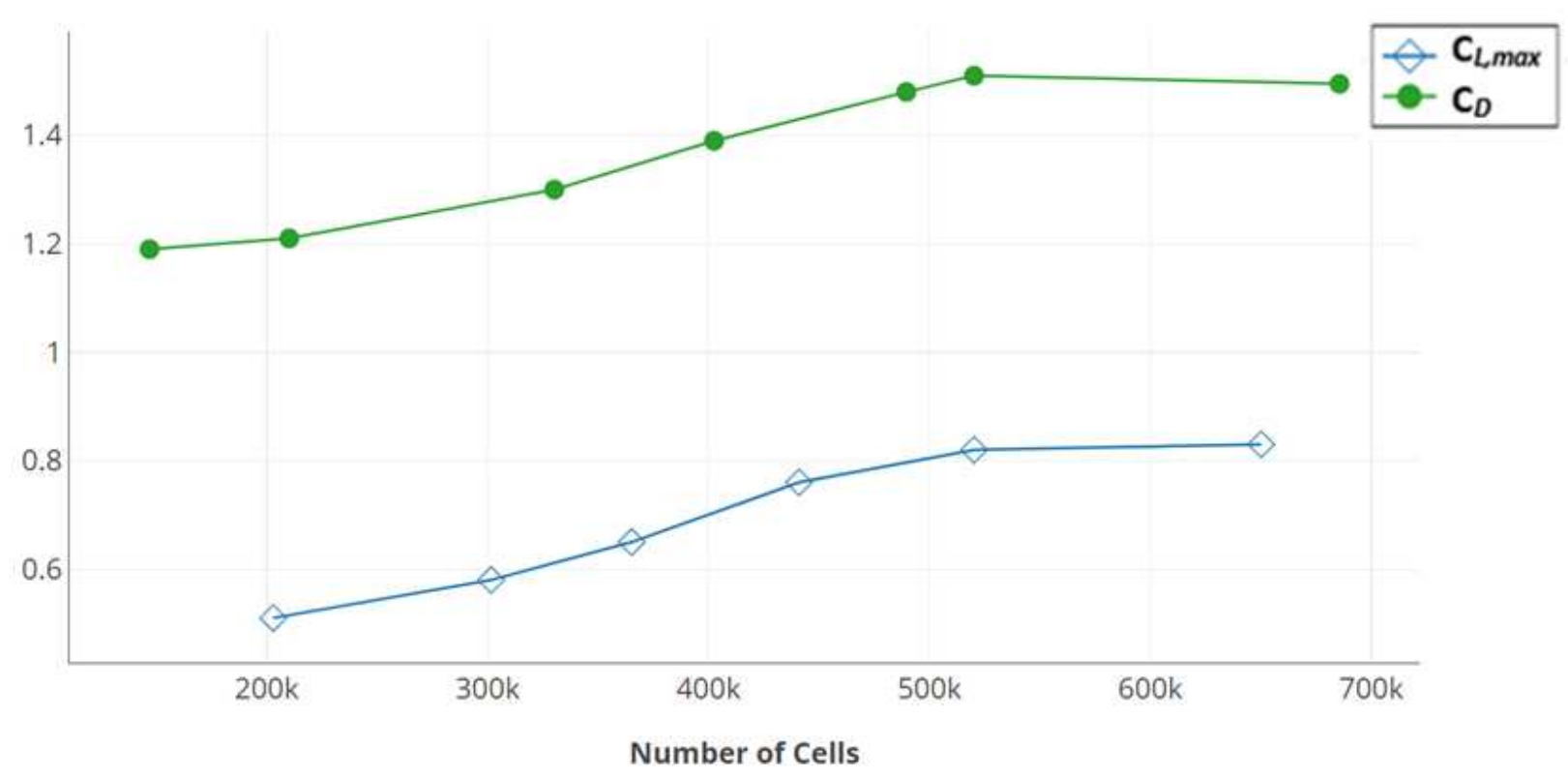

Fig. 11. Mesh dependency test results for the plain cylinder case.

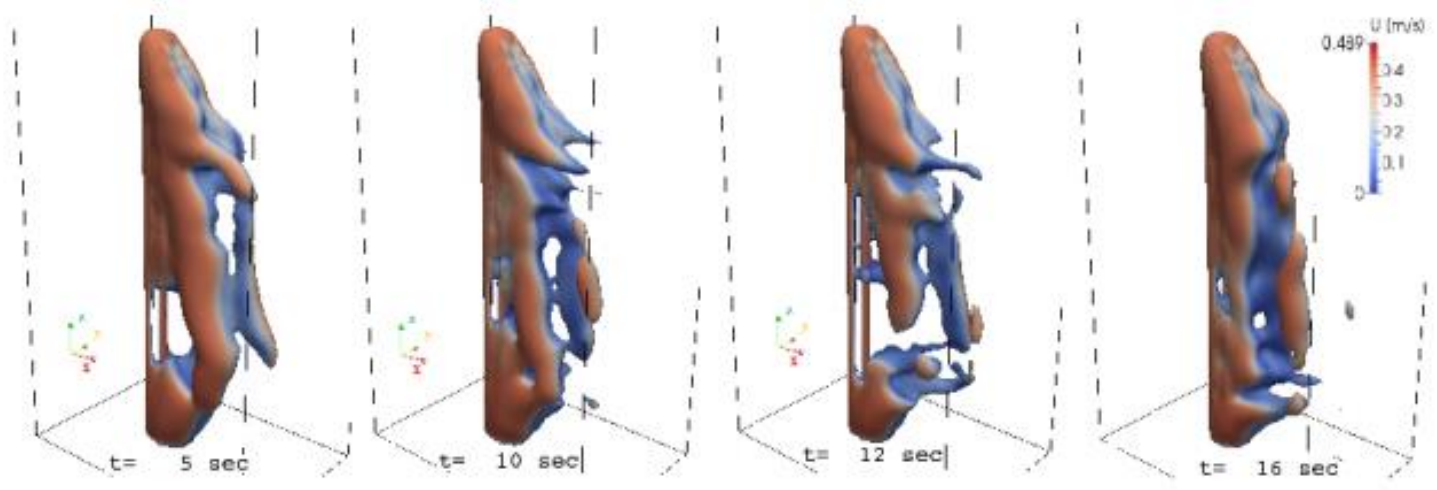

Fig. 12. Three-dimensional pressure contours in wake zone at different time steps and colored by velocity values for the bar cantilever case.

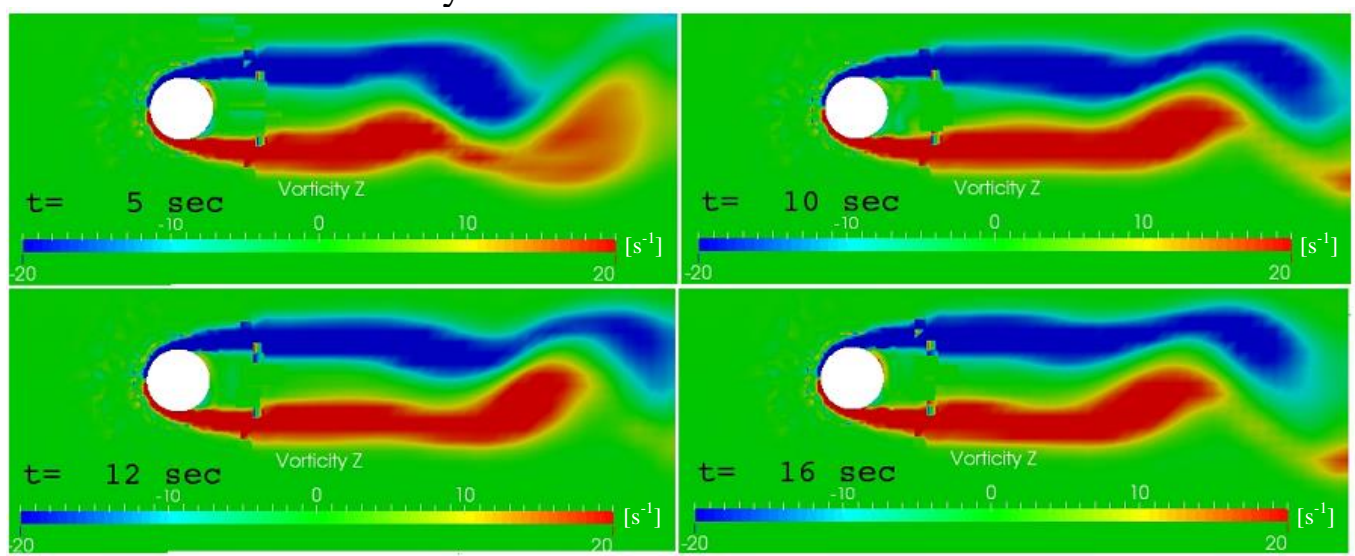

Fig. 13. Vorticity contours at $\mathrm{z}=0.2$ meters from the bottom wall at different time steps. 

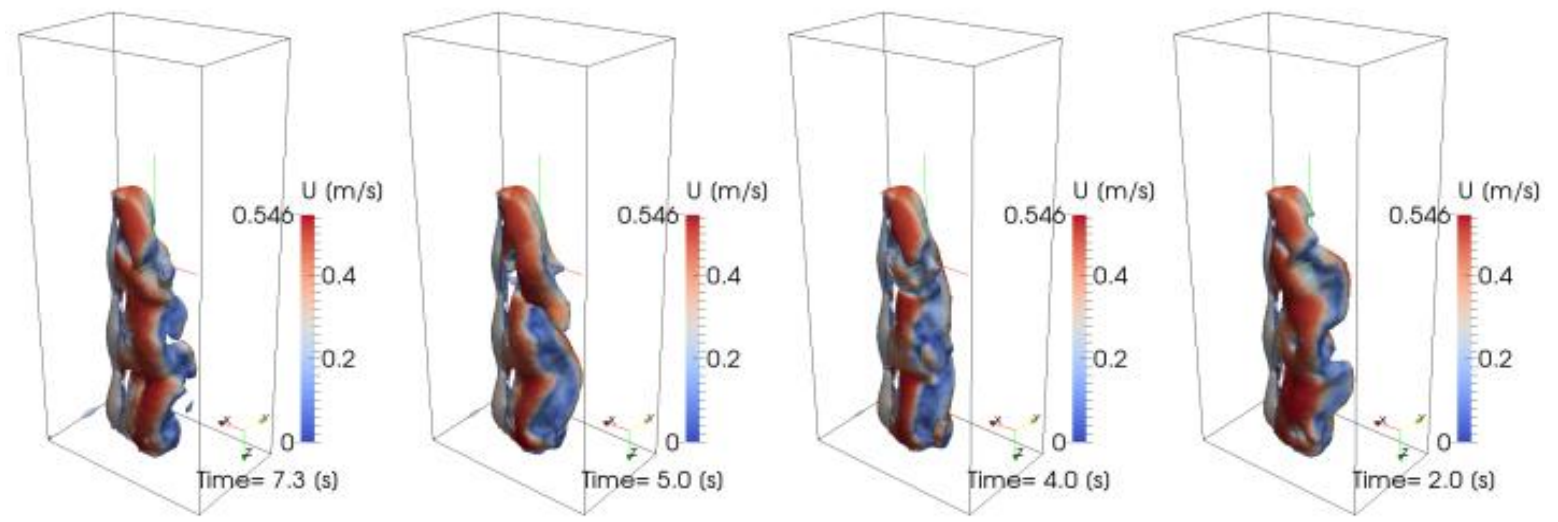

Fig. 14. Three-dimensional pressure contours in wake zone at different time steps and colored by velocity values for the straked cantilever case.
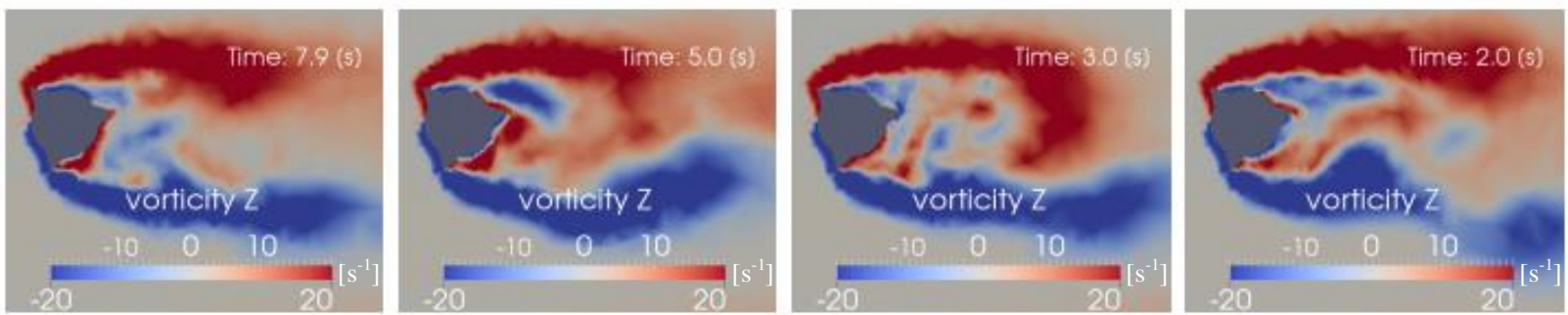

Fig. 15. Vorticity contours at $\mathrm{z}=0.2$ meters from the bottom wall at different time steps for the straked cantilever case.
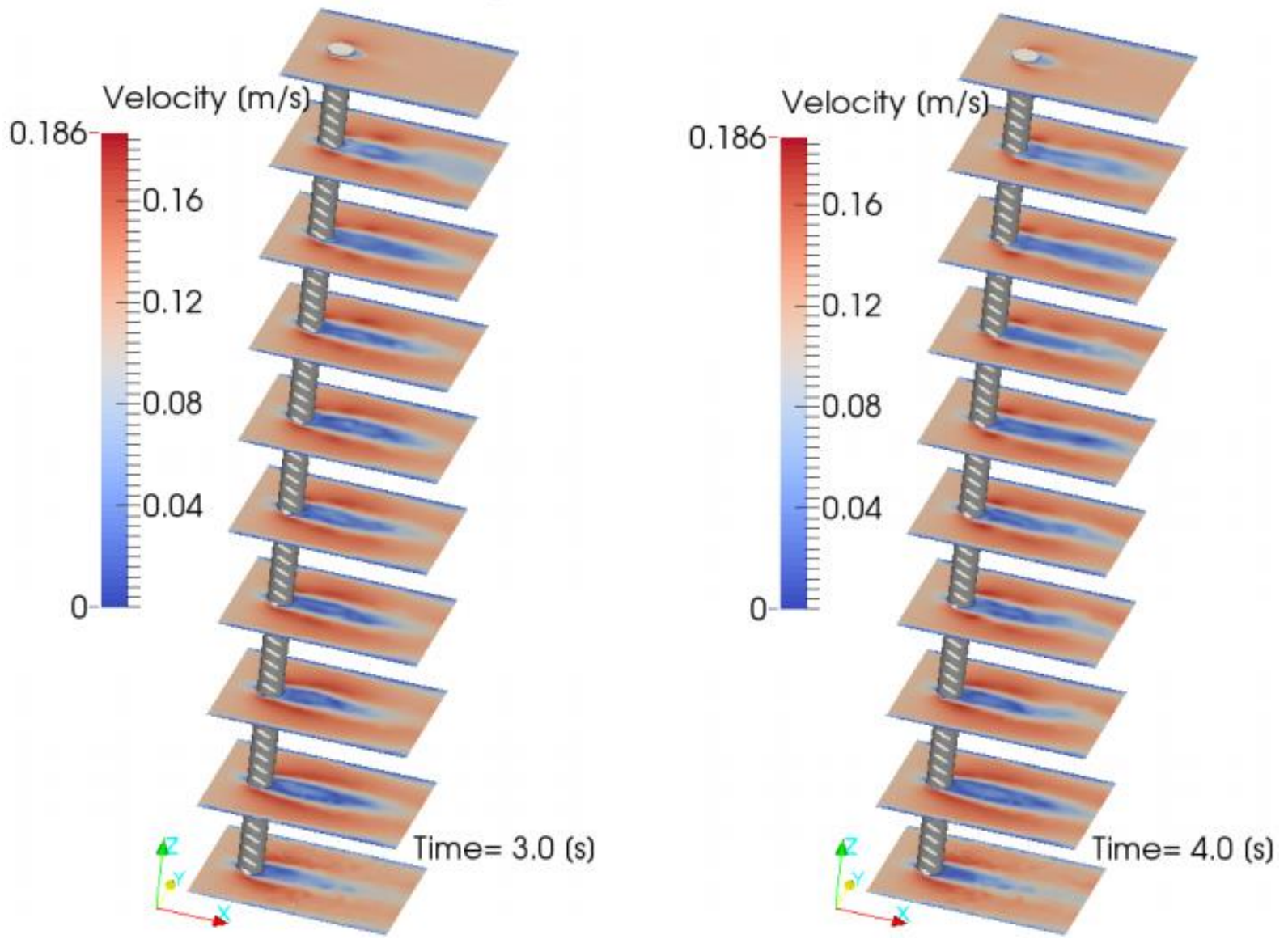


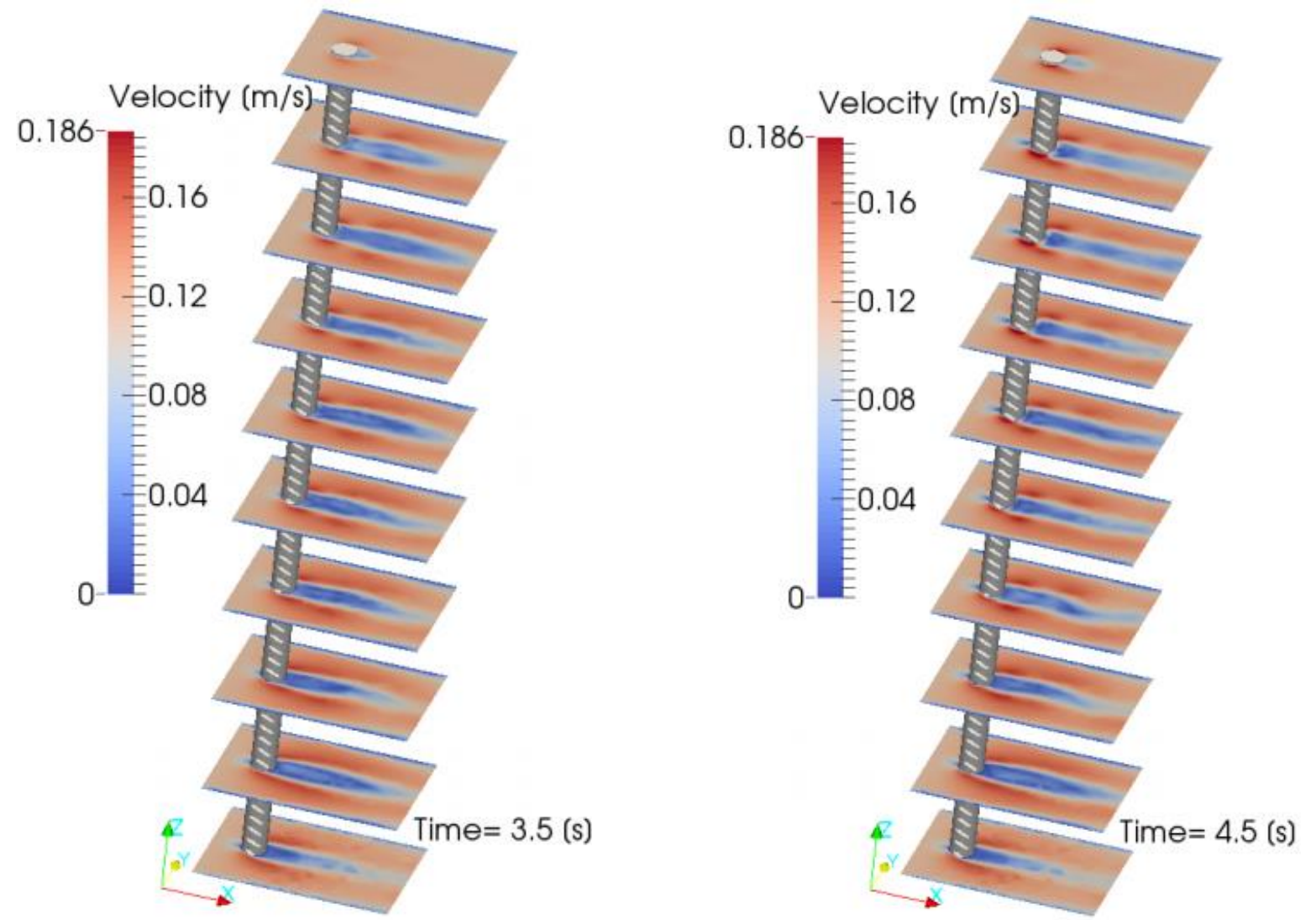

Fig. 16. The velocity results at different sections and time steps of the presented VIV suppression device. The sections are positioned at equal 9 distances alongwise. 

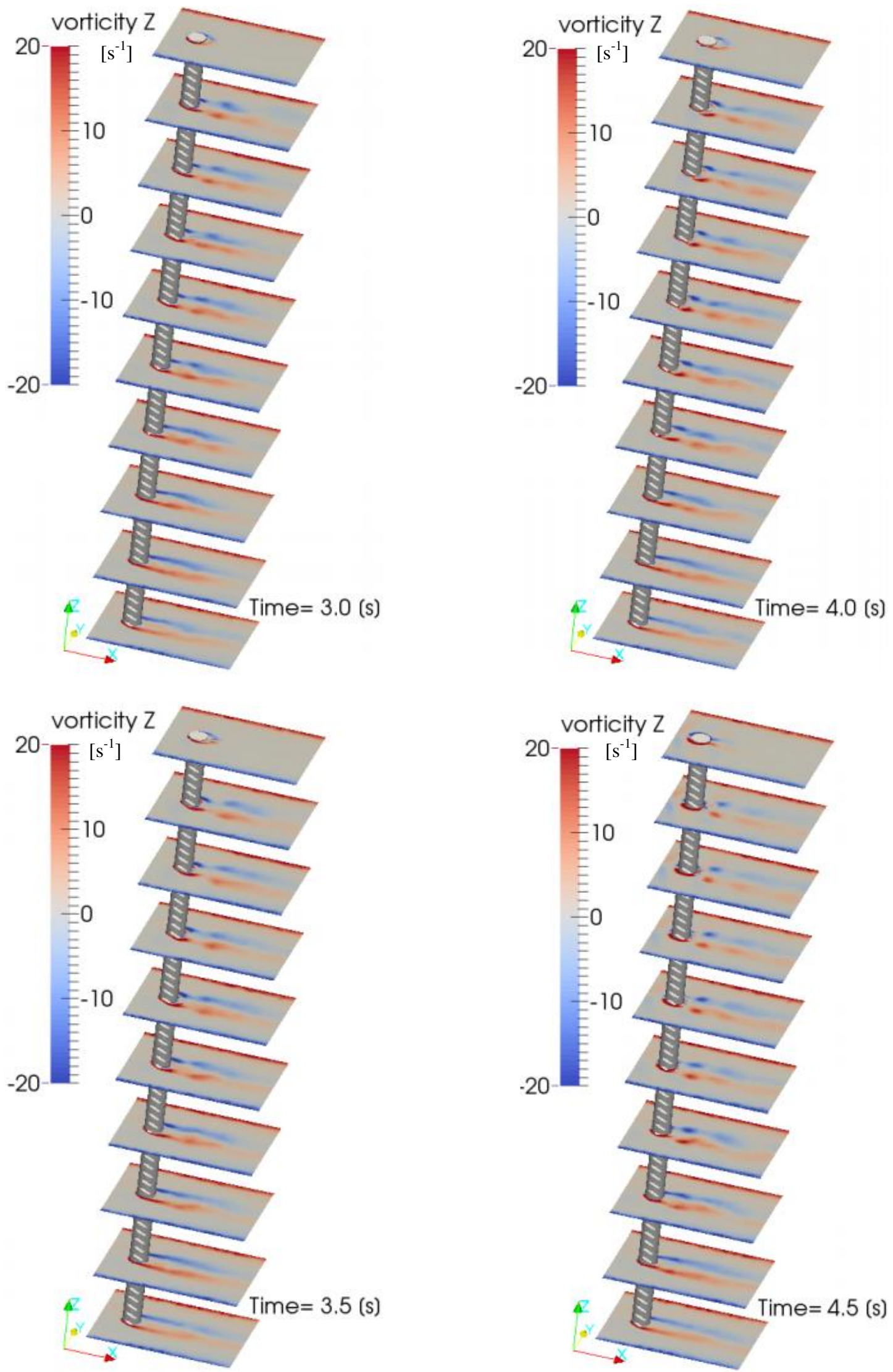

Fig. 17. The Z-vorticity results with different sections and time steps of the presented VIV 
suppression device. The sections are positioned at equal 9 distances alongwise.

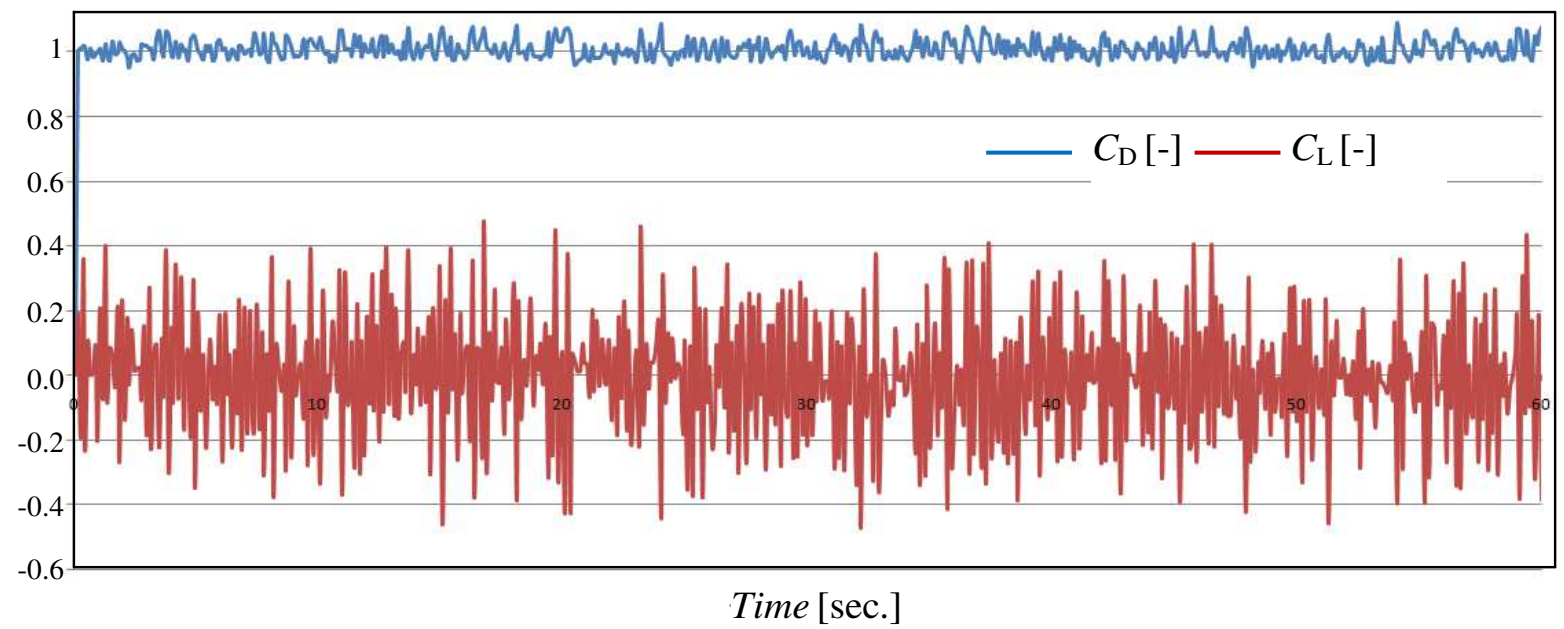

Fig. 18. Both lift and drag coefficients time history of the modified suppression device.

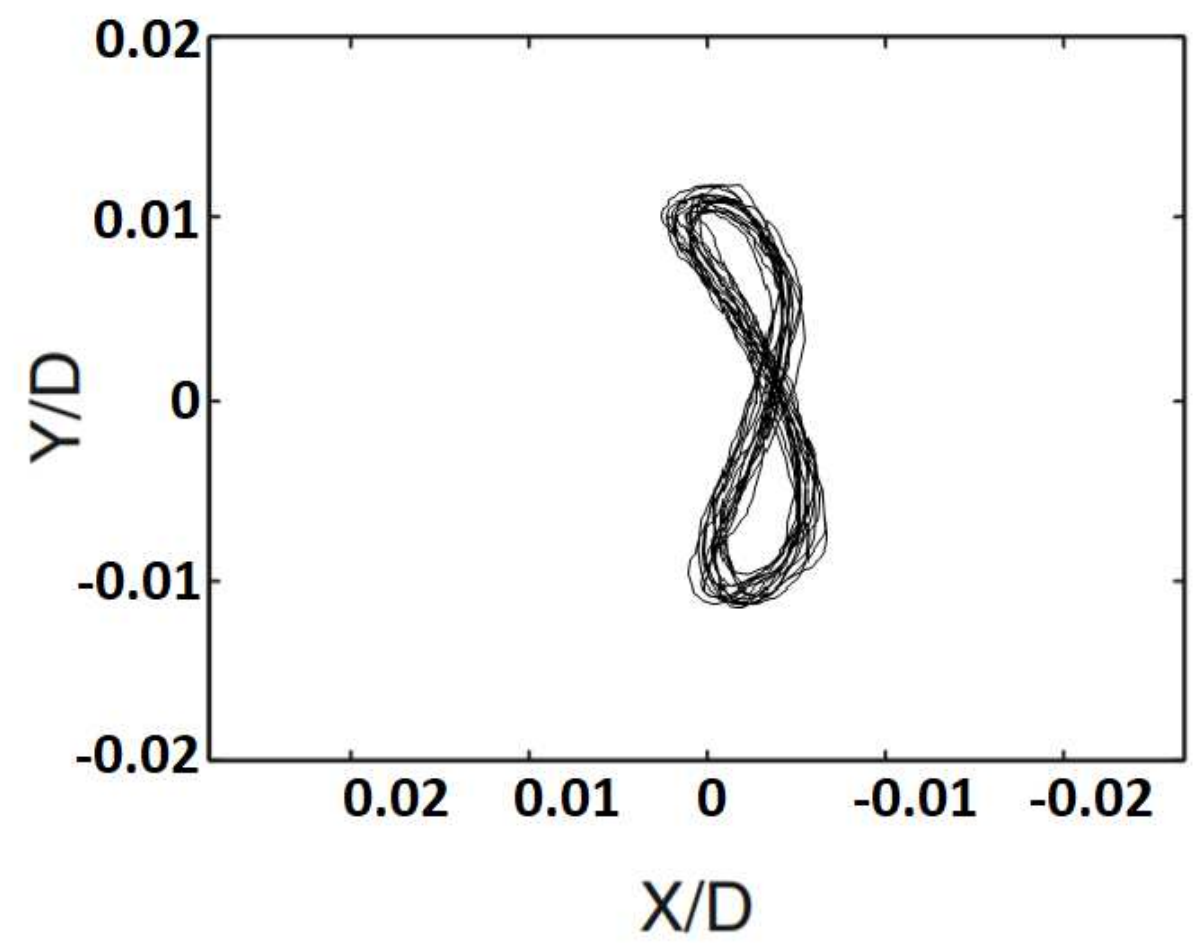

Fig. 19: Lissajous figure showing the $\mathrm{x}-\mathrm{y}$ trajectory of the flexible cylinder free tip of the shape two. 


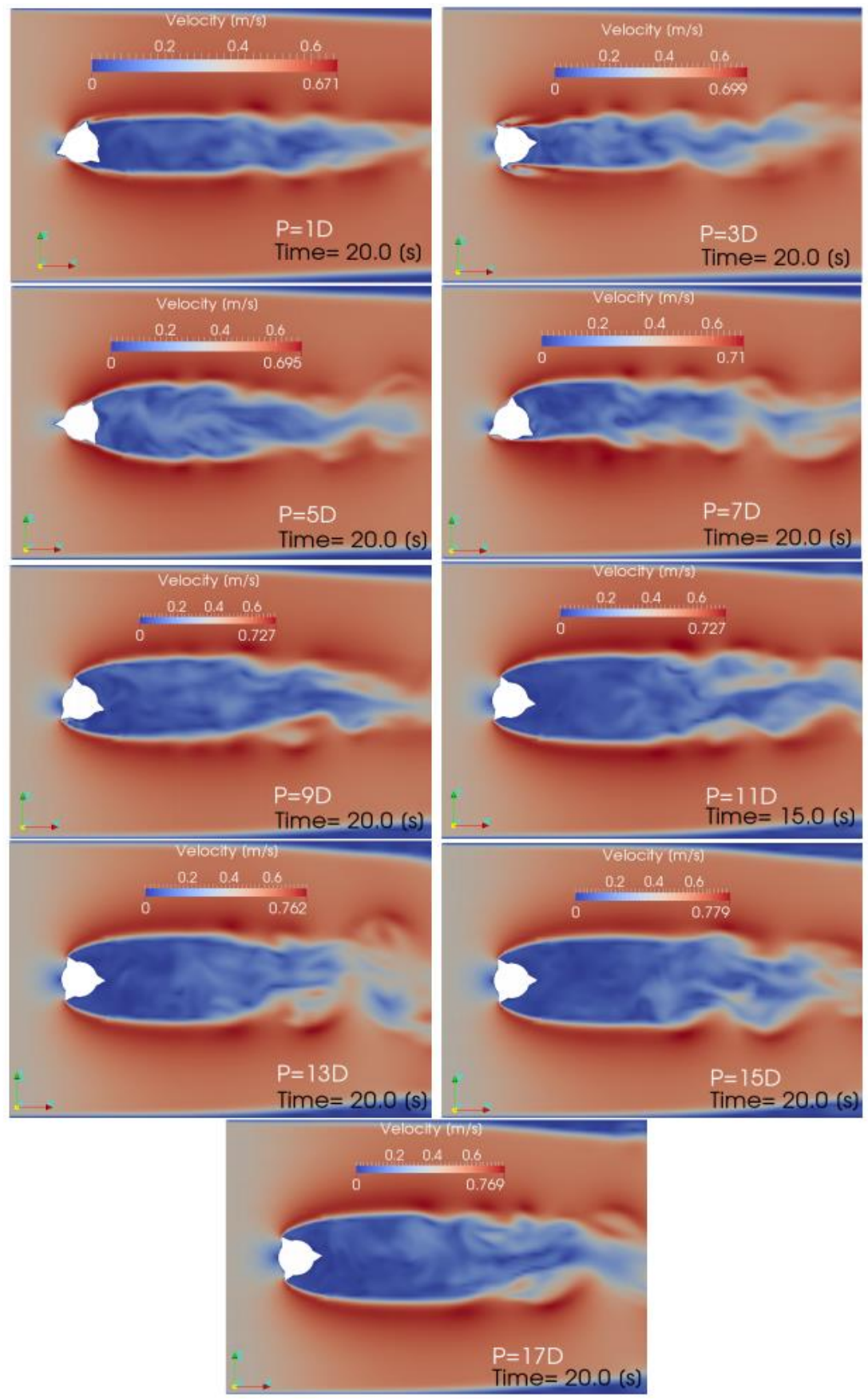

Fig. 20: The flow velocity contours at different stakes pitches taken at a slice on the mid point ydirection. 

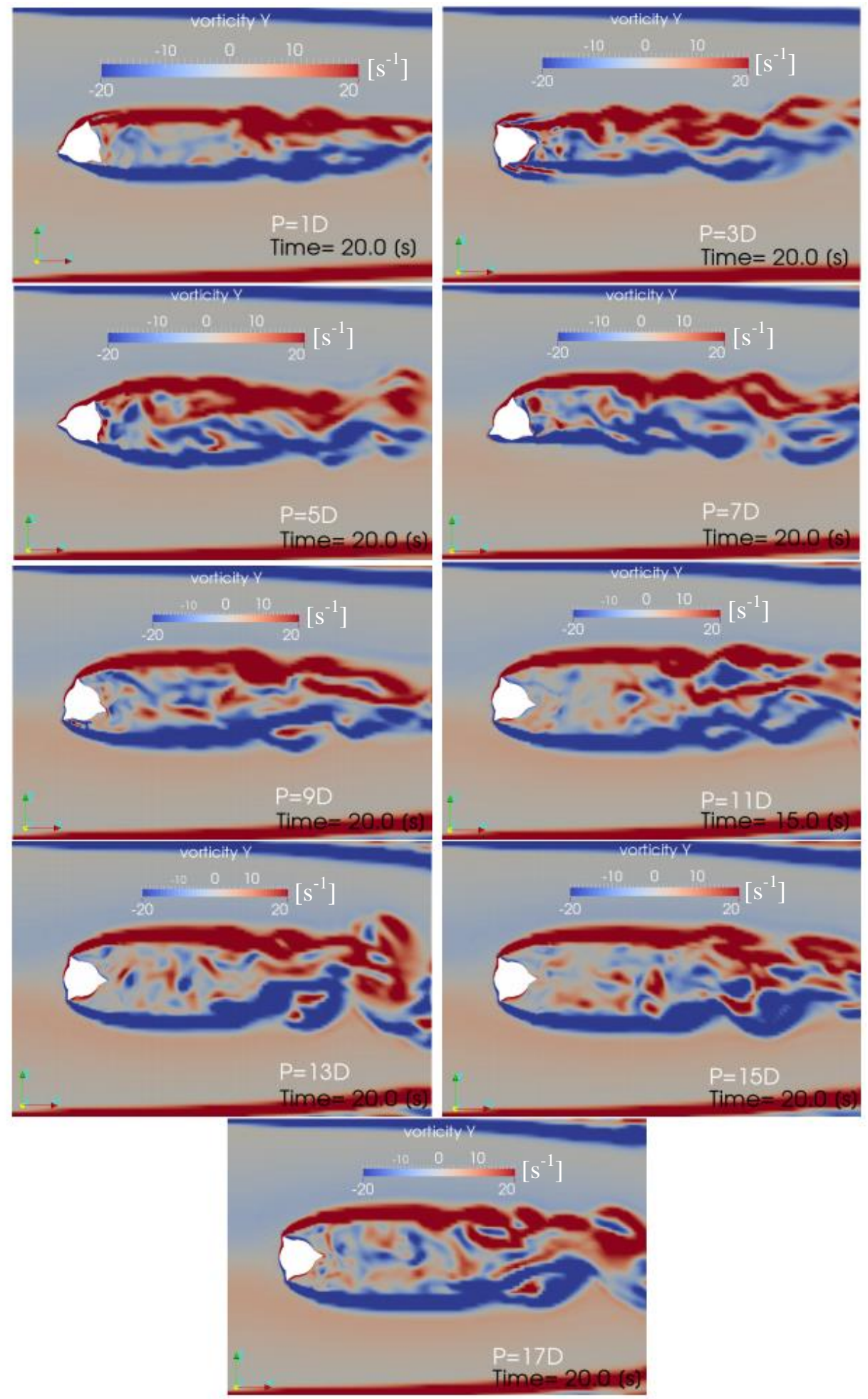

Fig. 21: The y-vorticity contours at different stakes pitches taken at a slice on the mid point ydirection. 


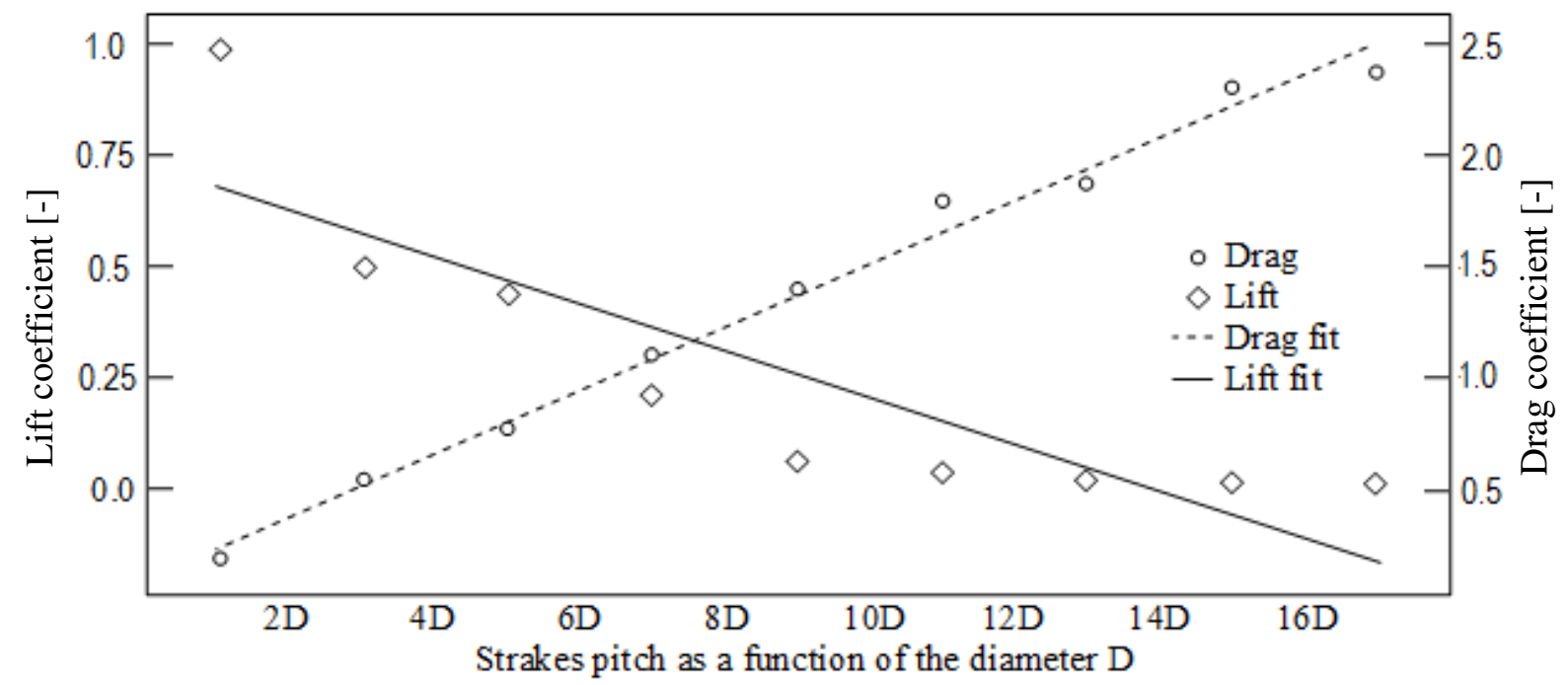

Fig. 22: The time averaged drag and lift forces with respect to stakes pitch.

Table (1): Comparison between the present solver and some of similar published solvers.

\begin{tabular}{clll}
\hline Refrence & \multicolumn{1}{c}{ Coupling } & Fluid & Solid \\
\hline Present work & Partitioned block G-S & FVM & FVM \\
\hline Habchi et al. [14] & Partitioned block G-S & FVM & FVM \\
\hline Kassiotis et al. [23] & Partitioned block G-S & FVM & FEM \\
\hline Vazquez [24] & Partitioned block G-S & FEM (stabilized) & FEM \\
\hline Mok [25] & Partitioned block G-S & FEM (stabilized) & FEM \\
\hline Gerbeau et al. [26] & Partitioned Quasi Newtonian & FEM (stabilized) & FEM \\
\hline
\end{tabular}

Where: G-S: Gauss-Seidel FVM: Finite Volume Method and FEM: Finite Element Method

Table (2): The ratio between the maximum displacement of the cantilever tip in $x$ and $y$ directions with respect to the reduced velocity.

\begin{tabular}{ccccc}
\hline \multirow{2}{*}{ Reduced velocity, $u^{*}$} & \multicolumn{2}{c}{$\mathrm{x} / \mathrm{D}$ ratio in $x$-direction } & \multicolumn{2}{c}{$\mathrm{y} / \mathrm{D}$ ratio in $y$-direction } \\
\cline { 2 - 5 } & Present work & Franzini [7] & Present work & Franzini [7] \\
\hline 3.69 & 0.115 & 0.11 & 0.32 & 0.31 \\
\hline 6.03 & 0.54 & 0.52 & 0.288 & 0.28 \\
\hline
\end{tabular}

Table (3): Strake shape designs. 


\begin{tabular}{lll}
\hline & Shape one & Shape two \\
\hline Number of strakes & 3 & 2 \\
\hline Strakes continuity & Continues & Start and break at equal distences \\
& & alongwise the riser at $6.35 \mathrm{~mm}$ \\
\hline Cross-section & Circular $\mathrm{D}=25.4 \mathrm{~mm}$ & Circular $\mathrm{D}=25.4 \mathrm{~mm}$ \\
\hline Hight of strakes & $4 \mathrm{~mm}$ & $4 \mathrm{~mm}$ \\
\hline
\end{tabular}

Table (4): The design and material parameters.

\begin{tabular}{cccccc}
\hline $\mathrm{S}(\mathrm{mm})$ & $\mathrm{D}(\mathrm{mm})$ & $\mathrm{L} / \mathrm{D}$ & $\mathrm{m}^{*}$ & $f_{1}(\mathrm{~Hz})$ & $\mathrm{EI} \mathrm{(Nm2)}$ \\
\hline 305 & 25.4 & 35.5 & 1.2 & 1.17 & 5.2 \\
\hline
\end{tabular}

Table (5): The results comparison for plain cylinder, strake one and strake two.

\begin{tabular}{cccc}
\hline Parameter & Plain cylinder & Shape One & Shape Two \\
\hline$C_{L, \text { max }}$ & 0.82 & 0.67 & 0.4 \\
\hline$C_{D}$ & 1.51 & 1.3 & 1.05 \\
\hline$y / D$ & 0.76 & 0.21 & 0.012 \\
\hline
\end{tabular}




\section{Supplementary Files}

This is a list of supplementary files associated with this preprint. Click to download.

- Conflictof Interest.docx

- Highlights.doc

- Coverletter.docx 